\title{
Calcium Secretion Coupling at Calyx of Held Governed by Nonuniform Channel-Vesicle Topography
}

\author{
Christoph J. Meinrenken, ${ }^{1}$ J. Gerard G. Borst, ${ }^{2}$ and Bert Sakmann ${ }^{1}$ \\ ${ }^{1}$ Max Planck Institute for Medical Research, 69120 Heidelberg, Germany, and 2Swammerdam Institute for Life Sciences, \\ University of Amsterdam, 1098 SM Amsterdam, The Netherlands
}

\begin{abstract}
Phasic transmitter release at synapses in the mammalian CNS is regulated by local $\left[\mathrm{Ca}^{2+}\right]$ transients, which control the fusion of readily releasable vesicles docked at active zones (AZs) in the presynaptic membrane. The time course and amplitude of these $\left[\mathrm{Ca}^{2+}\right]$ transients critically determine the time course and amplitude of the release and thus the frequency and amplitude tuning of the synaptic connection. As yet, the spatiotemporal nature of the $\left[\mathrm{Ca}^{2+}\right]$ transients and the number and location of release-controlling $\mathrm{Ca}^{2+}$ channels relative to the vesicles, the "topography" of the release sites, have remained elusive. We used a time-dependent model to simulate $\mathrm{Ca}^{2+}$ influx, threedimensional buffered $\mathrm{Ca}^{2+}$ diffusion, and the binding of $\mathrm{Ca}^{2+}$ to the release sensor. The parameters of the model were constrained by recent anatomical and biophysical data of the calyx of Held. Comparing the predictions of the model with previously
\end{abstract}

measured release probabilities under a variety of experimental conditions, we inferred which release site topography is likely to operate at the calyx: At each $\mathrm{AZ}$ one or a few clusters of $\mathrm{Ca}^{2+}$ channels control the release of the vesicles. The distance of a vesicle to the cluster(s) varies across the multiple release sites of a single calyx (ranging from 30 to $300 \mathrm{~nm}$; average $\sim 100 \mathrm{~nm}$ ). Assuming this topography, vesicles in different locations are exposed to different $\left[\mathrm{Ca}^{2+}\right]$ transients, with peak amplitudes ranging from 0.5 to $40 \mu \mathrm{M}$ (half-width $\sim 400 \mu \mathrm{sec}$ ) during an action potential. Consequently the vesicles have different release probabilities ranging from $<0.01$ to 1 . We demonstrate how this spatially heterogeneous release probability creates functional advantages for synaptic transmission.

Key words: active zone; buffer; diffusion; glutamate; heterogeneity; synapse; transmitter release; vesicle; domain
During fast synaptic transmission the release of neurotransmitter from vesicles in presynaptic terminals is controlled by calcium ions $\left(\mathrm{Ca}^{2+}\right)$ (Katz, 1969). Brief influx of $\mathrm{Ca}^{2+}$ through voltagegated channels causes a transient rise in the intracellular concentration of $\mathrm{Ca}^{2+}\left(\left[\mathrm{Ca}^{2+}\right]\right)$. Within $<1 \mathrm{msec}$ this rise in $\left[\mathrm{Ca}^{2+}\right]$ causes vesicles to fuse with the presynaptic membrane, releasing transmitter. The transient rise in $\left[\mathrm{Ca}^{2+}\right]$ is less pronounced with increasing distance from the $\mathrm{Ca}^{2+}$ channels. Therefore, a vesicle (i.e., the $\mathrm{Ca}^{2+}$ sensor controlling its release) must be located sufficiently close to one or more $\mathrm{Ca}^{2+}$ channels. The exact distance between channels and vesicles critically determines the time course and amplitude of the $\left[\mathrm{Ca}^{2+}\right]$ signal at the vesicles and thus determines the time course and amplitude of the release rate (Augustine and Neher, 1992). Therefore, understanding the functional and spatial organization of the release-controlling $\mathrm{Ca}^{2+}$ channels relative to vesicles (henceforth "topography of release sites") is crucial for a quantitative description of synaptic transmission itself (Augustine, 2001).

Because direct measurements of the local $\left[\mathrm{Ca}^{2+}\right]$ transients are

Received Aug. 31, 2001; revised Nov. 26, 2001; accepted Nov. 29, 2001

J.G.G.B. was supported by a Pioneer program of the Netherlands Organization for Scientific Research. We thank Erwin Neher for advice on the present work; Johann Heiner Bollmann, Ora Ohana, Arnd Roth, and Lisa D. Silverman for advice on this manuscript; Raphael J. Utz for the term "topography" as well as for late-night linguistic and diplomatic guidance; art for biomed/E. Heil (Frankfurt/M) for three-dimensional graphics (Fig. 8); and everyone in the Department of Cell Physiology at the Max Planck Institute for Medical Research, Heidelberg, for helpful discussions.

Correspondence should be addressed to Christoph Meinrenken, Max Planck Institute for Medical Research/4, Jahnstrasse 29, 69120 Heidelberg, Germany. E-mail: cjm@mpimf-heidelberg.mpg.de.

J. G. G. Borst's present address: Department of Neuroscience, Erasmus University, Rotterdam, The Netherlands.

Copyright $(\odot 2002$ Society for Neuroscience $0270-6474 / 02 / 221648-20 \$ 15.00 / 0$ not (yet) available, our understanding of the local $\left[\mathrm{Ca}^{2+}\right]$ dynamics during action potentials (APs) must rely on experimental data on release and on quantitative models (Neher, 1998a). A number of studies have investigated the significance of channel/vesicle location (Yamada and Zucker, 1992; Cooper et al., 1996; Gil et al., 2000), some of them quantifying release on the basis of measured $\mathrm{Ca}^{2+}$ sensitivity of the release-controlling $\mathrm{Ca}^{2+}$ sensor (Chow et al., 1994; Klingauf and Neher, 1997; Bennett et al., 2000). However, the considerable number of poorly known parameters in the models often has defeated attempts to derive with certainty the topography of release sites (Neher, 1998a).

We present a time-resolved model that simulates $\mathrm{Ca}^{2+}$ influx, three-dimensional buffered $\mathrm{Ca}^{2+}$ diffusion, and the binding of $\mathrm{Ca}^{2+}$ to the release sensor for the calyx of Held (henceforth, calyx), a giant terminal in the medial nucleus of the trapezoid body (MNTB) of mammalian brainstem (Forsythe et al., 1995). The model is based on recent anatomical and biophysical data, which constrain key parameters of the simulations. We infer the topography of release sites by comparing simulated transmitter release with previous experimental data (effects of added exogenous $\mathrm{Ca}^{2+}$ buffers BAPTA and EGTA as well as effects of altered $\mathrm{Ca}^{2+}$ channel gating). The topographic analysis indicates that the "readily releasable" pool of vesicles is heterogeneous with respect to its release probability. Heterogeneous release probability of vesicles has been observed at the calyx (Wu and Borst, 1999; Sakaba and Neher, 2001b). We show that, other than being an intrinsic property of the release apparatus, the heterogeneity may arise to a large extent from variability in the distances between vesicles and release-controlling $\mathrm{Ca}^{2+}$ channels at different release sites of a single calyx.

Our model reproduces the experimental data, including previ- 


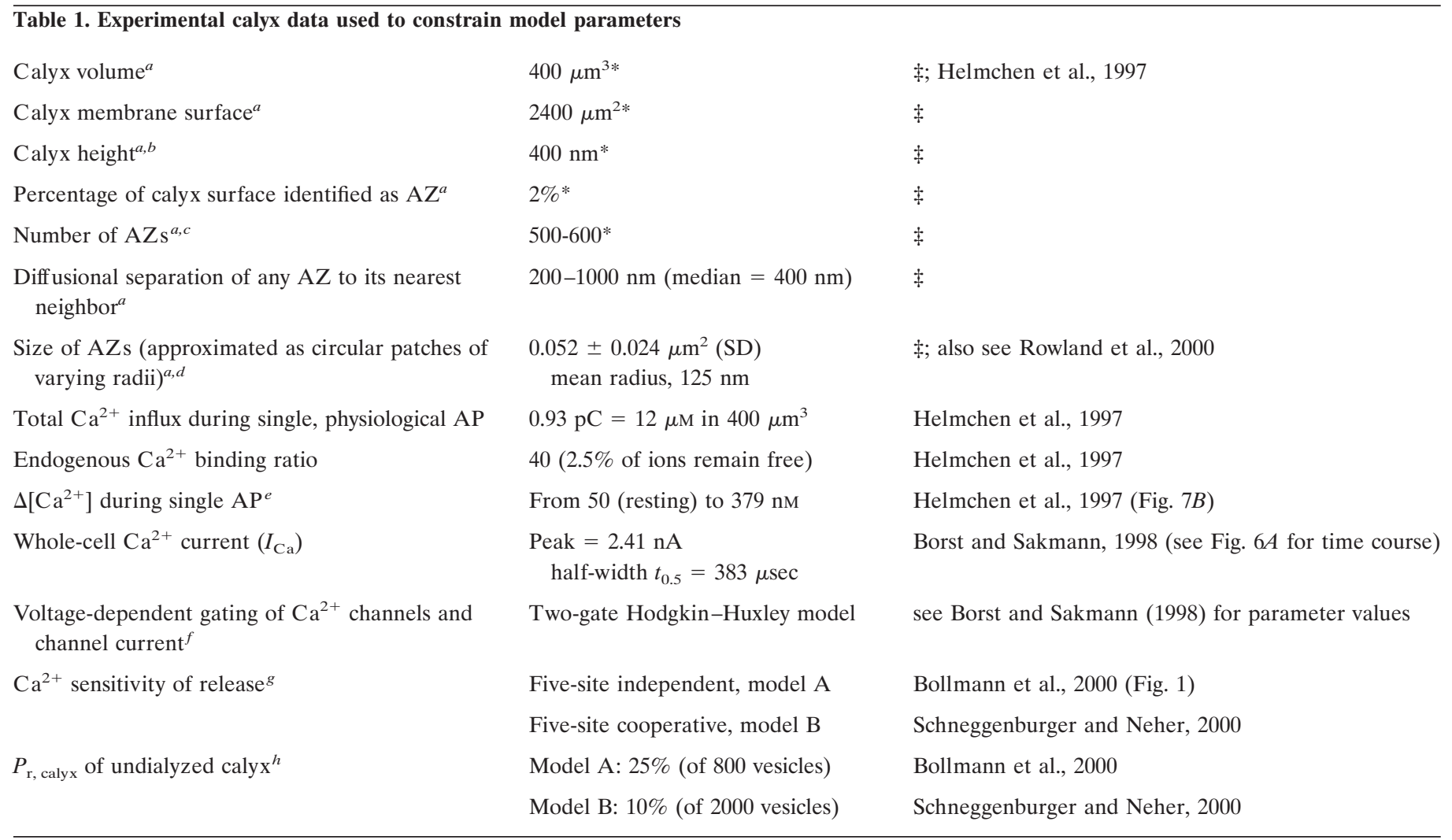

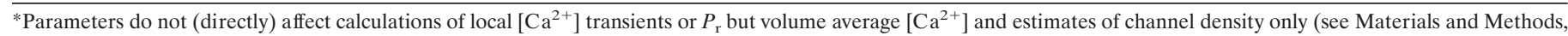
Discussion).

† Sätzler, Söhl, Bollmann, Borst, Frotscher, Sakmann, and Lübke, unpublished data.

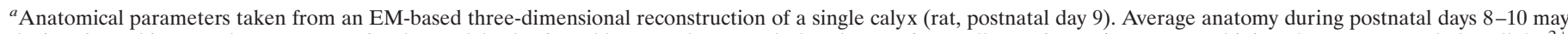

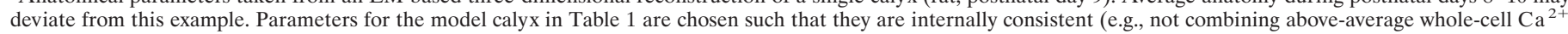
current with below-average calyx volume).

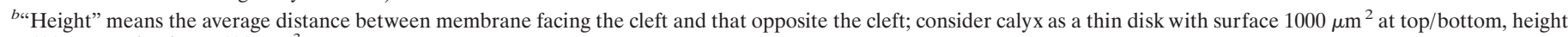
$\sim 400 \mathrm{~nm}$, and volume $400 \mu \mathrm{m}^{3}$.

${ }^{c}$ Corresponding to an average of 1.3 (Release Model A) or 3.3 (Release Model B) readily releasable vesicles docked per AZ.

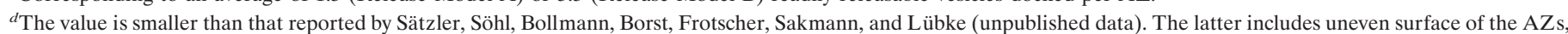
which does not contribute to diffusional distance.

${ }^{e} 379 \mathrm{nM}$ is the equilibrium $\left[\mathrm{Ca}^{2+}\right]$ after adding $12 \mu \mathrm{M}$ free $\mathrm{Ca}^{2+}$ to the calyx (with ATP and endogenous fixed buffer).

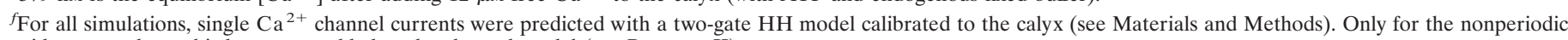
grid topography, a third gate was added to the channel model (see Property II).

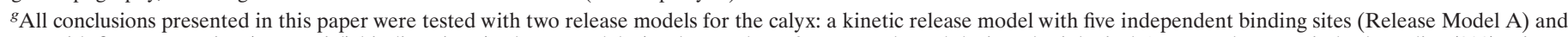

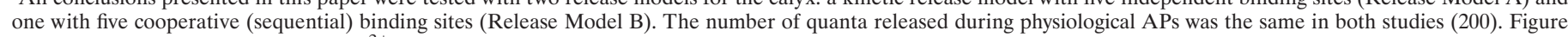
1 shows a direct comparison of the $\mathrm{Ca}^{2+}$ sensitivities of the models.

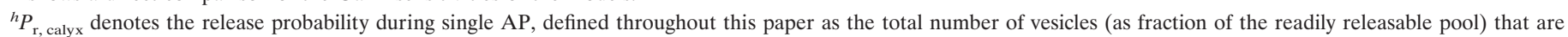
released in response to a single AP (phasic release only).

ously unexplained effects of exogenous $\mathrm{Ca}^{2+}$ buffers, only if the spatial nonuniformity is included explicitly in the calculations (discussed in Quastel et al., 1992). To demonstrate further the functional significance of the proposed nonuniformity, we investigated its effects on synaptic delay and on release during consecutive APs.

\section{MATERIALS AND METHODS}

Inferring the topography

The model simulates the time course $(0-5 \mathrm{msec}$ for a single AP; room temperature) of $\mathrm{Ca}^{2+}$ influx, three-dimensional buffered $\mathrm{Ca}^{2+}$ diff usion, and phasic transmitter release for a calyx at the developmental stage postnatal days $8-10$. Parameters in the simulations were constrained by electrophysiological and morphological measurements of the calyx (Table 1). The only remaining crucial but unknown parameters were the conductance of single $\mathrm{Ca}^{2+}$ channels and the channel-vesicle topography at release sites. We assumed a topography and then set the single $\mathrm{Ca}^{2+}$ channel conductance such that the predicted release probability for physiological conditions is the same as that observed in the experiments (see Results for values). We then simulated release under nonphysiological conditions: added exogenous $\mathrm{Ca}^{2+}$ buffers, lowered $\left[\mathrm{Ca}^{2+}\right]$ of the extracellular solution, and reduced open probability of $\mathrm{Ca}^{2+}$ channels (using, for each topography, the same single channel conductance as that used for the physiological condition). The predicted effects of the nonphysiological conditions on release critically depend on the assumed topography. Testing different hypothetical topographies, we compared the results of the model with the experimental data (Table 2) and thus inferred whether a particular topography is likely to be present at the calyx or not.

\section{Numerical simulations}

In the simulations the calyx volume was split up into subcompartments ("reaction volumes") of identical size around active zones (AZs; see Results for dimensions). For the periodic grid topography (see Results), boundary conditions (to adjoining compartments) for the buffered diffusion were periodic (side walls only). For all other simulations the boundaries were "closed," and the location of the channel cluster and the vesicles on the $\mathrm{AZ}$, the radius of the circular $\mathrm{AZ}$, as well as the stochastic $\mathrm{Ca}^{2+}$ currents through channels were different in each subcompartment 


\section{Table 2. Experimental calyx data used to infer topography of release sites}

\author{
0.2 mM EGTA: $25 \pm 2.5 \%(\mathrm{SEM})$ \\ 1 mM EGTA: $16.5 \pm 1.7 \%$ \\ 10 mM EGTA: $11.3 \pm 2.4 \%$ \\ 0.05 mM BAPTA: $25 \pm 3.8 \%(\mathrm{SEM})$ \\ 1 mM BAPTA: $8.6 \pm 0.9 \%$ \\ 10 mM BAPTA: $<1 \%^{b}$
}

$n=2.2-3.5$

Apparent Hill coefficient: $P_{\mathrm{r}, \text { calyx }} \propto\left(\int I_{\mathrm{Ca}} \cdot d t\right)^{n}$, when reducing $\left[\mathrm{Ca}^{2+}\right]_{\mathrm{ex}}{ }^{c}$

$P_{\mathrm{r}, \text { calyx }} \propto\left(\int I_{\mathrm{Ca}} \cdot d t\right)^{m}$, when reducing channel open probability by AP waveforms ${ }^{d}$

$P_{\mathrm{r}, \text { calyx }} \propto\left(\int I_{\mathrm{Ca}} \cdot d t\right)^{m}$, when partially blocking P/Qtype $\mathrm{Ca}^{2+}$ channels
Borst and Sakmann, 1996

Borst et al., 1995

(model results: Fig. 9A)

Barnes-Davies and Forsythe, 1995; Borst and Sakmann, 1999a; Wu et al., 1999 (model results: Fig. 9B)

Borst and Sakmann, 1999a (model results: Fig. $6 B$ )

Wu et al., 1999

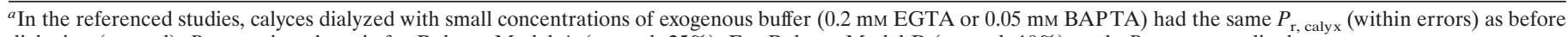
dialyzing (control). $P_{\mathrm{r} \text {, calyx }}$ given here is for Release Model A (control: $\left.25 \%\right)$. For Release Model B (control: $\left.10 \%\right)$, scale $P_{\mathrm{r} \text {, calyx }}$ accordingly.

${ }^{b}$ Implied phasic release probability during APs as estimated from Borst and Sakmann (1999b).

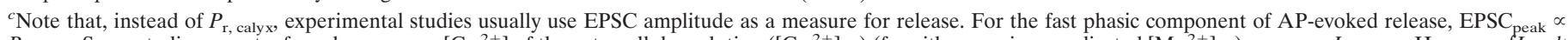

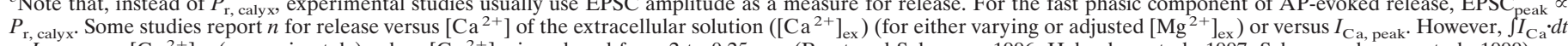

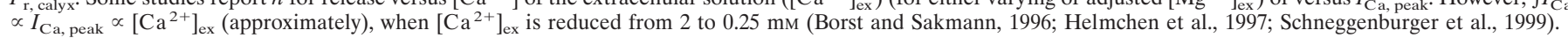

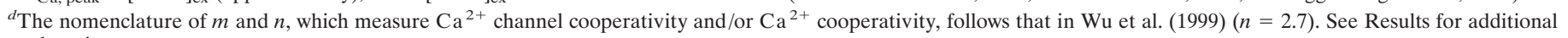
explanation.
}

(see Results). Dimensions of the compartments were chosen sufficiently large so that reflections of $\mathrm{Ca}^{2+}$ at the walls affect only volume average $\left[\mathrm{Ca}^{2+}\right]$ but not local $\left[\mathrm{Ca}^{2+}\right]$ near the vesicles (see below). The local $\left[\mathrm{Ca}^{2+}\right]$ transients around individual AZs in the model were assumed to be independent. The model was implemented as Ansi C code, running on a Silicon Graphics Oregon 2000 computer (processor MIPS RP1200, 300 $\mathrm{MHz})$. A single simulation $(0-5 \mathrm{msec})$ took $\sim 45 \mathrm{~min}$ to complete.

\section{Hodgkin-Huxley model for $\mathrm{Ca}^{2+}$ channel gating and time course of $\mathrm{I}_{\mathrm{Ca}}$}

To simulate $\mathrm{Ca}^{2+}$ influx through individual channels in response to APs, the model uses a two-gate Hodgkin-Huxley model with parameters that were fit for the calyx. The gates of the channels and the resulting currents are driven by AP waveforms (equations and parameters as in Borst and Sakmann, 1998). For some simulations with low channel open probability, a third gate was added (see Results). As the time course for $\mathrm{Ca}^{2+}$ entry at each channel location $\left[i_{\mathrm{Ca}}(t)\right]$, the simulations used either the "uniform $i_{\mathrm{Ca}}$ mode" or the "stochastic $i_{\mathrm{Ca}}$ mode." In the uniform $i_{\mathrm{Ca}}$ mode all channels are "open" and $i_{\mathrm{Ca}}(t)$ is the same for all channels, with a time course matching that of the whole-cell $\mathrm{Ca}^{2+}$ current (predicted by the Hodgkin-Huxley Model). In the stochastic $i_{\mathrm{Ca}}$ mode the model varies $i_{\mathrm{Ca}}(t)$ for each channel stochastically. In this mode the individual channel locations contribute different $i_{\mathrm{Ca}}(t)$, and some remain closed. Individual $i_{\mathrm{Ca}}(t)$ are simulated by using Monte Carlo-type pseudo-stochastic sampling to determine the open and closed times of the two gates [random number generator, $\operatorname{ran}(2)$ (Press et al., 1988); time step for $i_{\mathrm{Ca}}(t), 1 \mu \mathrm{sec}$ ]. Whenever a channel is open, the current is given according to the electrical driving force and the conductance. Single channel conductance was varied for different topographies (see above) but was the same for all channels. Note that values for single channel conductance given in Results refer to open channels, whereas values for channel current $i_{\mathrm{Ca}}$ [given as the peak amplitude of $i_{\mathrm{Ca}}(t), i_{\mathrm{Ca}}$, peak $]$ refer to the average across all open or closed channels. To simulate experiments with reduced $\left[\mathrm{Ca}^{2+}\right]$ of the extracellular solution, we reduced the channel conductance to match the reduction of whole-cell $I_{\mathrm{Ca}}$ observed in the experiments.

\section{Buffered diffusion of $\mathrm{Ca}^{2+}$}

At time $0, \mathrm{Ca}^{2+}$ and buffers were at resting concentrations and at spatial equilibrium. Standard equations (3.20-3.23 in Smith, 2001) for diff usion and buffering were solved numerically (forward Euler finite difference). We assumed unrestricted diff usion of $\mathrm{Ca}^{2+}$ and buffers around $\mathrm{AZs}$ (i.e., barriers, particularly nondocked vesicles in the vicinity of AZs, were neglected). To validate the unrestricted diffusion assumption (Glavinovic and Rabie, 2001) for the calyx, we analyzed the three-dimensional reconstruction of 31 of the $\sim 600$ AZs (K. Sätzler, L. Söhl, J. Bollmann,
J. Borst, M. Frotscher, B. Sakmann, and J. Lübke, unpublished data). In a dome-like control volume around each AZ (200 nm distance from the edge of the control volume to the nearest point on AZ), there were, on average, 62 vesicles (not docked, i.e., not readily releasable). These vesicles occupied $\sim 6 \%$ of the dome-like control volume. Therefore, their effect as diff usion barriers is negligible.

Only for the simulation of consecutive APs (see Results), a linear extrusion mechanism was used, which reduces the $\left[\mathrm{Ca}^{2+}\right]$ of each voxel separately by $\left(\left[\mathrm{Ca}^{2+}\right]-\left[\mathrm{Ca}^{2+}\right]_{\text {rest }}\right) \cdot \gamma \cdot \Delta t$ per time step, where $\left[\mathrm{Ca}^{2+}\right]_{\text {rest }}$ $=50 \mathrm{~nm}$ (see below), $\gamma=400 \mathrm{~Hz}$ is the pump rate, and $\Delta t$ is the time step.

Spatial resolution was as follows: $(5 \mathrm{~nm})^{3}$ voxels for the first six layers on the membrane $(0-30 \mathrm{~nm}),(10 \mathrm{~nm})^{3}$ for $30-90 \mathrm{~nm}$, and $(20 \mathrm{~nm})^{3}$ for the remainder. After $1.5 \mathrm{msec}$, when $\left[\mathrm{Ca}^{2+}\right]$ gradients have dissipated, spatial resolution was decreased by collapsing neighboring voxels into (10 $\mathrm{nm})^{3},(20 \mathrm{~nm})^{3}$, and $(40 \mathrm{~nm})^{3}$. For those voxels (of the first layer) located "above" the presumed $\mathrm{Ca}^{2+}$ channels, $\Delta\left[\mathrm{Ca}^{2+}\right]=i_{\mathrm{Ca}}(t) \cdot \Delta t /(V \cdot \mathrm{F})$ per time step was added to $\left[\mathrm{Ca}^{2+}\right]$ of the voxel $(\Delta t$, time step; $V$, voxel volume; F, Faraday's constant). Time step was $10 \mathrm{fsec}$ to $0.68 \mathrm{nsec}$, such that the relative change in concentration of any substance in any voxel during any time step did not exceed $\pm 1 \%$ (autoadaptive); $d t_{\max }=$ $0.15 \cdot(d x)^{2} / 220 \mu \mathrm{m}^{2} / \mathrm{sec}$, where $d x=5 \mathrm{~nm}$ for times up to $1.5 \mathrm{msec}$ and 10 nm thereafter.

Unless otherwise indicated, the model solution contained (control condition): free $\mathrm{Ca}^{2+}$ at a starting concentration of $\left[\mathrm{Ca}^{2+}\right]_{\text {rest }}=50 \mathrm{nM}$ (Helmchen et al., 1997) and diffusion coefficient $D_{\mathrm{Ca}}=220 \mu \mathrm{m}^{2} / \mathrm{sec}$ (Albritton et al., 1992); endogenous fixed buffer (termed EFB, unspecified identity, single $\mathrm{Ca}^{2+}$ binding site) with a binding ratio of 40 (Helmchen et al., 1997), total concentration $[\mathrm{EFB}]_{\text {total }}=80 \mu \mathrm{M}$, affinity $K_{\mathrm{D}}=$ $2 \mu \mathrm{M}$ (varied in sensitivity analyses between $200 \mathrm{nM}$ and $200 \mu \mathrm{M}$; see below), and a forward $\mathrm{Ca}^{2+}$ binding rate, $k_{\mathrm{on}}=5 \cdot 10^{8}$ per Msec (Klingauf and Neher, 1997) (sensitivity analysis below); ATP with $[\mathrm{ATP}]_{\text {total }}=0.58$ $\mathrm{mM}, K_{\mathrm{D}, \mathrm{Ca}}=200 \mu \mathrm{M}, k_{\mathrm{on}, \mathrm{Ca}}=5 \cdot 10^{8}$ per Msec (Baylor and Hollingworth, 1998) (with $k_{\mathrm{on}}$, Ca corrected for temperature; sensitivity analysis below), $D_{\text {ATP }}=220 \mu \mathrm{m}^{2} / \mathrm{sec}$. Kinetic parameters of ATP are for the binding of ATP to $\mathrm{Ca}^{2+}$ only (not $\mathrm{Mg}^{2+}$ ). The presence of $4 \mathrm{mM}$ Mg-ATP in the pipette during the experiments (Borst et al., 1995) was accounted for by reducing the concentration of total ATP available for $\mathrm{Ca}^{2+}$ binding to $0.58 \mathrm{~mm}$. The remaining ATP was assumed to stay bound to $\mathrm{Mg}^{2+}$ during the $\left[\mathrm{Ca}^{2+}\right]$ transient $\left(K_{\mathrm{D}, \mathrm{Mg}}=100 \mu \mathrm{M}\right)$ and thus unavailable for $\mathrm{Ca}^{2+}$ buffering (slow off-rate of $\mathrm{Mg}$-ATP, $k_{\text {off, }} \mathrm{Mg}=$ $150-390 / \mathrm{sec}$; Baylor and Hollingworth, 1998). For some simulations, mobile exogenous buffers were added at varying concentrations: BAPTA $\left(k_{\text {on }}=4 \cdot 10^{8}\right.$ per Msec, $K_{\mathrm{D}}=220 \mathrm{nM}, D_{\text {BAPTA }}=220 \mu \mathrm{m}^{2} / \mathrm{sec} ;$ Naraghi and Neher, 1997) and EGTA. The binding kinetics of EGTA are strongly $\mathrm{pH}$-dependent. We thus used two sets of parameters: "EGTA" $\left(k_{\mathrm{on}}=\right.$ 
$10 \cdot 10^{6}$ per Msec, $K_{\mathrm{D}}=70 \mathrm{~nm}$; Nägerl et al., 2000) or "EGTA-2" $\left(k_{\mathrm{on}}=\right.$ $2.5 \cdot 10^{6}$ per Msec, $K_{\mathrm{D}}=180 \mathrm{nM}$; Naraghi and Neher, 1997). $D_{\text {EGTA }}=$ $D_{\text {EGTA-2 }}=220 \mu \mathrm{m}^{2} / \mathrm{sec}$.

Because the buffered diffusion algorithm uses neither the steady-state assumption nor the rapid buffer or the linearized buffer approximation, it correctly simulates any local/global depletion of unbound buffers ("buffer saturation"; Naraghi and Neher, 1997). Because $\left[\mathrm{Ca}^{2+}\right]$ in the simulations is generally low (micromolar range), mobile buffers deplete only marginally. For example, in the simulation with added $1 \mathrm{~mm}$ BAPTA (see Fig. $7 D$ ), at the time of peak $\mathrm{Ca}^{2+}$ influx and at the center of the channel cluster (where depletion is the strongest), the concentration of unbound BAPTA is still $90 \%$ of the volume average concentration (94\% for unbound ATP). In contrast, unbound endogenous fixed buffer (EFB) is depleted locally because it binds $\mathrm{Ca}^{2+}$ without being replenished by diffusion. As a result, unbound EFB (at the cluster center and at the peak of the $\mathrm{Ca}^{2+}$ current) is depleted to $6 \%$ of the volume average concentration.

\section{$\mathrm{Net} \mathrm{Ca}^{2+}$ influx into calyx volume versus modeled subcompartments}

In the reference topography simulating physiological conditions with Release Model A, the conductance was $14.52 \mathrm{pS}$ per channel cluster, corresponding to an average $I_{\mathrm{Ca} \text {, peak }}=0.66 \mathrm{pA}$ per cluster. (The conductance for the simulations with the less $\mathrm{Ca}^{2+}$-sensitive Release Model $\mathrm{B}$ was $37.04 \mathrm{pS}$.) This corresponds to $0.26 \mathrm{fC}$ or $12 \mu \mathrm{M}$ unbuffered $\mathrm{Ca}^{2+}$ entering each subcompartment (volume, $0.110 \mu \mathrm{m}^{3}$ ), thus increasing volume average $\left[\mathrm{Ca}^{2+}\right]$ to $379 \mathrm{nM}$, as observed in experiments (see Table 1). The 600 subcompartments (for $600 \mathrm{AZs}$ ) contributed a total of 0.16 pC Ca ${ }^{2+}$ (per AP and assuming one cluster per AZ), which is $17 \%$ [42\% in case of Release Model B] of the whole-cell value observed in experiments (see Table 1). The total modeled volume (600 subcompartments or $\sim 17 \%$ of $400 \mu \mathrm{m}^{3}$ ) corresponds to this ratio. Therefore, the increase in volume average $\left[\mathrm{Ca}^{2+}\right]$ in the subcompartments was the same as that experimentally observed for the whole calyx. This approach indirectly accounts for $\mathrm{Ca}^{2+}$ that enters through channels located away from release sites, assuming that these channels do not (significantly) affect local $\left[\mathrm{Ca}^{2+}\right]$ transients at release sites but only the volume average $\left[\mathrm{Ca}^{2+}\right]$ (see Discussion). To comply with the experimental measurement of volume average $\left[\mathrm{Ca}^{2+}\right], \Delta\left[\mathrm{Ca}^{2+}\right]$ was $12 \mu \mathrm{M}$ in every simulation; whenever $\mathrm{Ca}^{2+}$ influx for the control condition was changed (for topographies other than the reference topography and for Release Model B; see Results), the volume of the subcompartments was adjusted accordingly. [The model does not include contributions to $\left[\mathrm{Ca}^{2+}\right]$ from the release of $\mathrm{Ca}^{2+}$ from intracellular stores. At the calyx this contribution during a single AP is marginal at most (Helmchen et al., 1997).]

\section{Release}

We defined release probability $P_{\mathrm{r}}$ (as a percentage) as the fraction of all readily releasable vesicles that are released during a single AP (phasic release only; see Table 1 for size of readily releasable pool). "Release site" is defined as the functional entity of one readily releasable vesicle and the one or more $\mathrm{Ca}^{2+}$ channels controlling its release. At the calyx $(\sim 600 \mathrm{AZs})$ a single $\mathrm{AZ}$ contains, on average, more than one release site (Sätzler, Söhl, Bollmann, Borst, Frotscher, Sakmann, and Lübke, unpublished data). Although different release sites at the same AZ may be controlled by the same $\mathrm{Ca}^{2+}$ channels, the model assumes that release from individual sites is stochastically independent. Under this assumption more than one vesicle during a single AP may be released from a single AZ (Auger and Marty, 2000; Sun and Wu, 2001). However, a single release site can release at most one vesicle per AP (the model does not include recovery of the readily releasable pool). Because it is a relative measure, predicted $P_{\mathrm{r}}$ does not depend on the total number of vesicles in the readily releasable pool (= number of release sites) nor on the number of AZs or the number of release sites per AZ.

To quantify $P_{\mathrm{r}}$ in response to a transient increase in $\left[\mathrm{Ca}^{2+}\right]$, the model uses, alternately, two kinetic schemes (Fig. 1): Release Model A (Bollmann et al., 2000) or Release Model B (Schneggenburger and Neher, 2000). Note that, because it is less $\mathrm{Ca}^{2+}$-sensitive, Release Model B predicts higher absolute $\left[\mathrm{Ca}^{2+}\right]$ transients than Release Model A. However, relative spatial profiles of the transients are almost identical (marginal depletion of mobile buffers; see above). Therefore, the conclusions on the release site topography are valid for either release model. For clarity, figures generally show $\left[\mathrm{Ca}^{2+}\right]$ transients and $P_{\mathrm{r}}$ of simulations with Release Model A. Analogous results with Release Model B are given in the text only. At time 0 , the $\mathrm{Ca}^{2+}$ binding sites of the sensors were equilibrated with $\left[\mathrm{Ca}^{2+}\right]_{\text {rest }}$. The readily releasable pool was "full." For each assumed vesicle location (location at which the membrane of the vesicle is closest to presynaptic membrane), the time course of the local $\left[\mathrm{Ca}^{2+}\right]$ transient predicted by the reaction-diffusion scheme (measured in the voxel $\sim 10 \mathrm{~nm}$ above the presynaptic membrane) was translated into a release rate versus time. This was done for each vesicle individually, assuming that (1) release sites are independent and (2) binding of $\mathrm{Ca}^{2+}$ to the release-controlling $\mathrm{Ca}^{2+}$ sensor does not affect $\left[\mathrm{Ca}^{2+}\right]$ (Yamada and Zucker, 1992). The differential equations of the model describing the relative state occupancies were solved numerically (forward Euler finite difference), using a variable time step such that, during any time step, the absolute change of the relative occupancy of any state was at most $\pm 0.5 \%$. The time integral $(0-5 \mathrm{msec})$ of the release rate of an individual vesicle is $P_{\mathrm{r}, \text { vesicle }}$. The (heterogeneous) release rates of individual vesicles were averaged into an average release rate versus time. The time integral of this rate $(0-5 \mathrm{msec})$ is the predicted average release probability of all vesicles in the calyx $\left(P_{\mathrm{r} \text {, calyx }}\right)$. Different release site topographies predict different $P_{\mathrm{r} \text {, calyx }}$ because they correspond to different distributions of channel-to-vesicle distances for the readily releasable pool. The average release rate was converted to an EPSC, as described by Bollmann et al. (2000) (convolution of release rate with quantal EPSC).

\section{Sensitivity of $\mathrm{P}_{r}$ to kinetics and concentrations of endogenous buffers}

The model includes the mobile $\mathrm{Ca}^{2+}$ buffer ATP as well as one fixed buffer. The fixed buffer simulates the effect of one or more not further identified fixed or poorly mobile buffers. We varied concentration and binding kinetics of the endogenous buffers to estimate the sensitivity of predicted $P_{\mathrm{r}}$ to these parameters. Even drastic variations in the parameters of endogenous buffers (two to three orders of magnitude) do not change the predicted $P_{\mathrm{r}, \text { calyx }}$ to a degree that would compromise our results on release site topography. The reference simulation for each parameter variation is the simulation for the reference topography (see Results; control condition, $P_{\mathrm{r} \text {, calyx }}=25 \%$ ).

Endogenous fixed buffer. We varied [EFB] between 10 and 10,000\% of the reference value $(80 \mu \mathrm{M})$ while adjusting $K_{\mathrm{D} \text {, EFB }}$ such that [EFB]/ $K_{\mathrm{D} \text {, EFB }}$ (approximate binding ratio) was kept unchanged at 40 . This variation changes the predicted $P_{\mathrm{r} \text {, calyx }}$ between 41 and $18 \%$, respectively. During an AP the EFB locally depletes/equilibrates with $\left[\mathrm{Ca}^{2+}\right]$ (see above) and thus is rendered ineffective as a local sink for $\mathrm{Ca}^{2+}$. Therefore, EFB has only a small effect on the direct attenuation of the local $\left[\mathrm{Ca}^{2+}\right]$ transients that control phasic release of transmitter. This shows that the predictions of the model of $P_{\mathrm{r}}$ are accurate even if the concentration of EFB in the presynaptic volume were not spatially uniform.

ATP. Two sets of parameter variations for ATP were investigated. (1) Keeping $[\mathrm{ATP}] / K_{\mathrm{D}, \mathrm{ATP}}$ constant, we changed both $[\mathrm{ATP}]$ and $K_{\mathrm{D} \text {, ATP }}$ between 10 and $1000 \%$ of their respective reference values (see above).

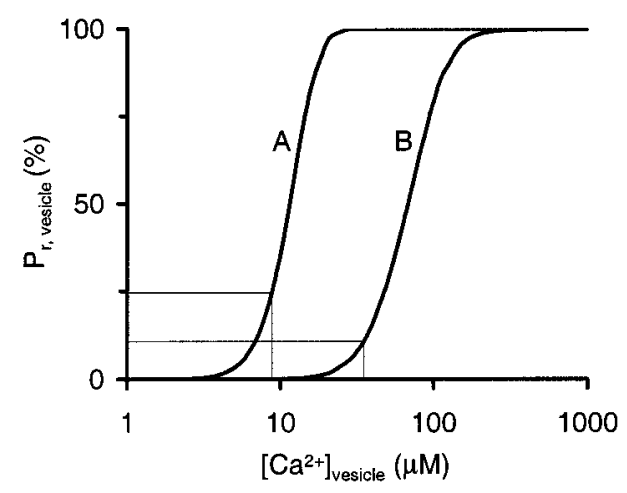

Figure 1. Intrinsic $\mathrm{Ca}^{2+}$ sensitivity of transmitter release. Shown is $P_{\mathrm{r}}$ for a single vesicle when exposed to a $\left[\mathrm{Ca}^{2+}\right]$ transient with a time course equal to that of whole-cell $I_{\mathrm{Ca}}$ (full width at half-maximum, $383 \mu \mathrm{sec}$ ) and with a peak amplitude of $\left[\mathrm{Ca}^{2+}\right]_{\text {vesicle. The thin lines indicate release }}$ probability during APs according to Release Model A $\left(P_{\mathrm{r}, \text { vesicle }}=25 \%\right.$ at $\left[\mathrm{Ca}^{2+}\right]_{\text {vesicle }}=8.8 \mu \mathrm{M}$; Bollmann et al., 2000) or Release Model B $\left(P_{\mathrm{r}, \text { vesicle }}=10 \%\right.$ at $\left[\mathrm{Ca}^{2+}\right]_{\text {vesicle }}=35 \mu \mathrm{M}$; Schneggenburger and Neher, 2000). 


\begin{tabular}{|c|c|c|c|}
\hline $\begin{array}{l}\text { Single versus } \\
\text { multiple channels } \\
\text { per vesicle }\end{array}$ & $\begin{array}{l}\text { Distance of } \\
\text { vesicle from } \\
\mathrm{Ca}^{2+} \text { source }\end{array}$ & $\begin{array}{l}\text { Consistent with } \\
\text { effects of } \\
\text { BAPTA and } \\
\text { EGTA on } P_{\mathrm{r}}\end{array}$ & $\begin{array}{l}\text { Consistent with } \\
\text { apparent Hill } \\
\text { coefficient } \\
m \sim n\end{array}$ \\
\hline \multirow[t]{2}{*}{ Single channel } & Fixed & - & - \\
\hline & Variable & + & - \\
\hline \multirow[t]{2}{*}{ Channel grid } & Narrow grid & - & + \\
\hline & Wide grid & + & - \\
\hline \multirow[t]{2}{*}{ Channel cluster(s) } & Fixed & - & + \\
\hline & Variable & + & + \\
\hline
\end{tabular}

This changes $P_{\mathrm{r} \text {, calyx }}$ between 37 and $22 \%$, respectively. (2) Keeping $[\mathrm{ATP}] \cdot k_{\mathrm{on}}$ (buffer) product constant, we changed both [ATP] and (inversely) $k_{\text {on, ATP }}$, between 10 and $1000 \%$ of the reference values. This changes $P_{\mathrm{r}, \text { calyx }}$ between $44 \%$ (low [ATP], high $k_{\text {on, ATP }}$ ) and $12 \%$ (low $k_{\text {on }}$, ATP , high [ATP]). Complete removal of ATP from the model calyx results in $P_{\mathrm{r}, \text { calyx }}=48 \%$. For simulations with the added exogenous buffers EGTA or BAPTA, the presence of ATP changes the predicted $P_{\mathrm{r}, \text { calyx }}$ even less because the effect of ATP is small compared with that of the exogenous buffer (see Fig. 4D).

\section{RESULTS}

In the first part of Results, we infer which channel-vesicle topography characterizes release sites at the calyx (developmental stage postnatal days 8-10). In the second part, we simulate the spatiotemporal pattern of AP-evoked $\left[\mathrm{Ca}^{2+}\right]$ transients and of phasic transmitter release at the calyx. In the third part, we illustrate the functional significance of the proposed release site topography for synaptic transmission at this fast synapse.

\section{Topography of release sites}

\section{Overview}

Because the location of $\mathrm{Ca}^{2+}$ channels at the calyx is not known, there is a multitude of conceivable topographic arrangements of channels relative to readily releasable vesicles. We analyzed various topographies for their compatibility with measured phasic release probabilities $\left(P_{\mathrm{r}}\right)$ under different experimental conditions (Table 3): (1) reducing the $\left[\mathrm{Ca}^{2+}\right]$ transients by dialyzing the calyx with exogenous $\mathrm{Ca}^{2+}$ buffers EGTA or BAPTA and (2) reducing the $\mathrm{Ca}^{2+}$ influx by altering the gating of the $\mathrm{Ca}^{2+}$ channels.

(1) The efficacy of $\mathrm{Ca}^{2+}$ buffers in reducing the $\left[\mathrm{Ca}^{2+}\right]$ transient around a $\mathrm{Ca}^{2+}$ channel depends on the diffusion distance from the channel (Neher, 1986). For the calyx it was found previously that there is no channel-to-vesicle distance, for which the theoretical efficacy of BAPTA versus EGTA is consistent with experimental data (Naraghi and Neher, 1997). Although we confirm this result, we find further that the observed buffer efficacies can be explained by assuming that the channel-to-vesicle distance is different for different release sites of the same calyx.

(2) When the open probability of $\mathrm{Ca}^{2+}$ channels at the calyx is reduced, $P_{\mathrm{r}}$ is reduced in a supralinear manner. On the basis of this experimental finding, it was concluded previously that the majority of readily releasable vesicles at the calyx is controlled by more than one $\mathrm{Ca}^{2+}$ channel per vesicle (Borst and Sakmann, 1999a). We find further that the multiple $\mathrm{Ca}^{2+}$ channels controlling a vesicle, rather than being distributed evenly in the membrane, are likely to be organized in clusters of $\mathrm{Ca}^{2+}$ channels.

On the basis of these findings, we suggest that phasic transmitter release at the calyx is governed by the following nonuniform topography of release sites (henceforth "reference topography").
Readily releasable vesicles are controlled by clusters of $\mathrm{Ca}^{2+}$ channels, with one or a few clusters per AZ. For any one release site the distances between the vesicle and the individual channels of a cluster are similar. However, vesicles at different release sites are located at a broad range of distances from the channel cluster (average distance $\sim 100 \mathrm{~nm}$; coefficient of variation $>0.5$ ). Details of the findings on topography are explained below as Properties I-III.

Property I: The distance between a vesicle and its releasecontrolling $\mathrm{Ca}^{2+}$ channel(s) varies across different release sites of the same calyx

For the calyx the distance between a vesicle and the $\mathrm{Ca}^{2+}$ channel(s) controlling its release is not known. Knowing the sensitivity of the $\mathrm{Ca}^{2+}$ sensor does not solve this problem, because the conductance of $\mathrm{Ca}^{2+}$ channels in the calyx is unknown. The $\left[\mathrm{Ca}^{2+}\right]$ transient that drives vesicle release could be supplied by a channel at distance $D$ and with conductance $C$ or, alternatively, from a channel twice as far away but with approximately twice the conductance (see below). This scaling behavior prevents one from inferring at what distances from $\mathrm{Ca}^{2+}$ channels the vesicles are likely to be located. However, the scaling is broken when experimental data on the effects of added exogenous $\mathrm{Ca}^{2+}$ buffers EGTA or BAPTA are taken into account.

When a calyx is loaded with EGTA or BAPTA, $P_{\mathrm{r}}$ is reduced in a concentration-dependent manner. For example, $10 \mathrm{~mm}$ EGTA reduces $P_{\mathrm{r}}$ to $\sim 0.45$ of $P_{\mathrm{r}}$ in the native calyx. BAPTA (1 $\mathrm{mM}$ ), which binds $\mathrm{Ca}^{2+}$ faster than EGTA, reduces $P_{\mathrm{r}}$ to $\sim 0.35$ of $P_{\mathrm{r}}$ in the native calyx (see Table 2). The reduction of $P_{\mathrm{r}}$ by the buffers is ascribed to the effect of the buffers on $\left[\mathrm{Ca}^{2+}\right]$ transients. EGTA and BAPTA intercept part of the $\mathrm{Ca}^{2+}$ diff using from the inner mouth of the channels to the vesicles, thereby reducing the peak amplitude of the $\left[\mathrm{Ca}^{2+}\right]$ transients reaching the vesicles $\left(\left[\mathrm{Ca}^{2+}\right]_{\text {vesicle }}\right)$. In combination with experimental data, diffusion calculations thus may be used to infer characteristic distances between release-controlling $\mathrm{Ca}^{2+}$ channels and vesicles. This has been addressed previously for the calyx (Naraghi and Neher, 1997). Here we confirm the earlier result while offering a different interpretation.

We begin by assuming that every releasable vesicle at an AZ in the calyx is located at some fixed distance from a single $\mathrm{Ca}^{2+}$ channel that controls its release (e.g., $80 \mathrm{~nm})($ Fig. $2 A)$. The $\mathrm{Ca}^{2+}$ current through each channel gives rise to a $\left[\mathrm{Ca}^{2+}\right]$ transient, with a peak amplitude decaying rapidly with increasing distance from the channel. Therefore, to yield similar $\left[\mathrm{Ca}^{2+}\right]_{\text {vesicle }}$ (and thus $P_{\mathrm{r}}$ ) for different vesicle distances, we increased the single channel conductance for increasing distance (Fig. 2B). Single channel conductance was chosen such that the predicted release probability for a vesicle $\left(P_{\mathrm{r} \text {, vesicle }}\right)$ was $25 \%$ under control conditions ("control" means that the model calyx contained only endogenous fixed buffer and ATP). For any given distance the simulations for added buffers used the same channel conductance as that used for the control condition. The time course of the single channel current $\left[i_{\mathrm{Ca}}(t)\right.$; approximately Gaussian] was calculated with a Hodgkin-Huxley Model. In these simulations $i_{\mathrm{Ca}}(t)$ was the same for all channels (uniform $i_{\mathrm{Ca}}$ mode; see Materials and Methods). Depending on the conductance, peaks of $i_{\mathrm{Ca}}$ (per channel) varied from $0.0157 \mathrm{pA}$ (for distance of $5 \mathrm{~nm}$ ) to 0.936 (for distance of $125 \mathrm{~nm}$ ). After the addition of exogenous buffers, the predicted $\left[\mathrm{Ca}^{2+}\right]_{\text {vesicle }}$ is strongly dependent on distance (Fig. 2C). This is expected because, for a single $\mathrm{Ca}^{2+}$ channel domain (Neher, 1986), the reduction in $\left[\mathrm{Ca}^{2+}\right]_{\text {vesicle }}$ 
A
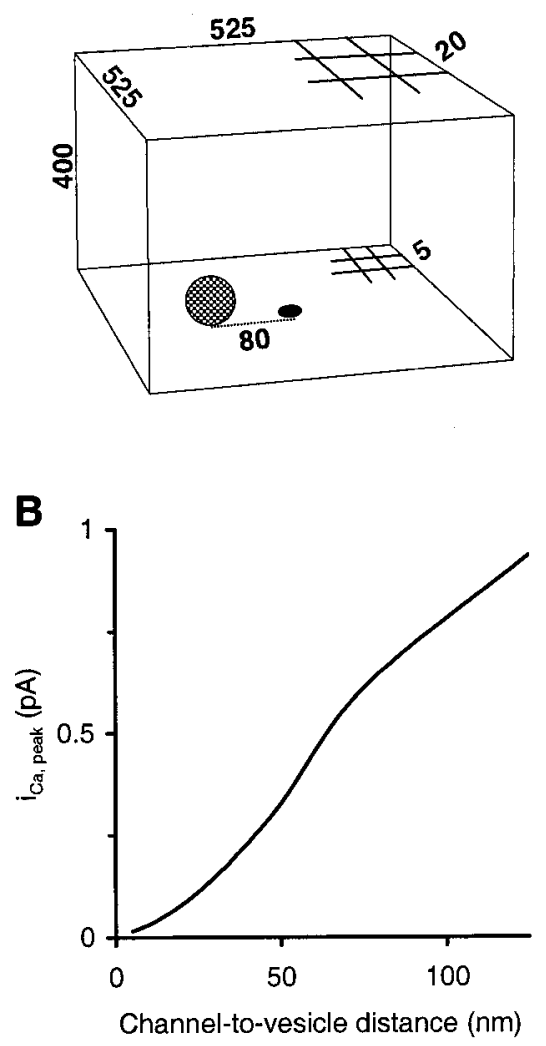

C

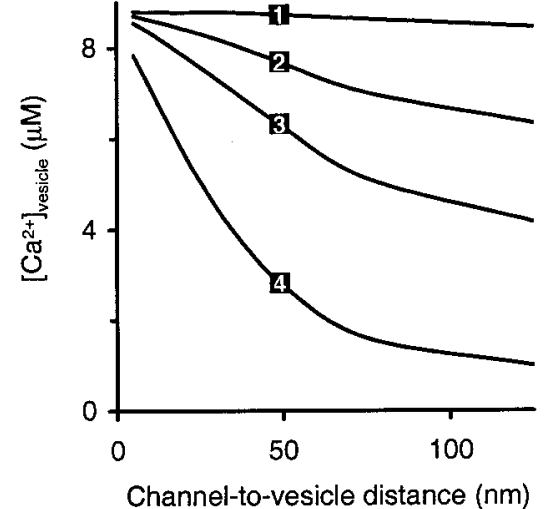

D

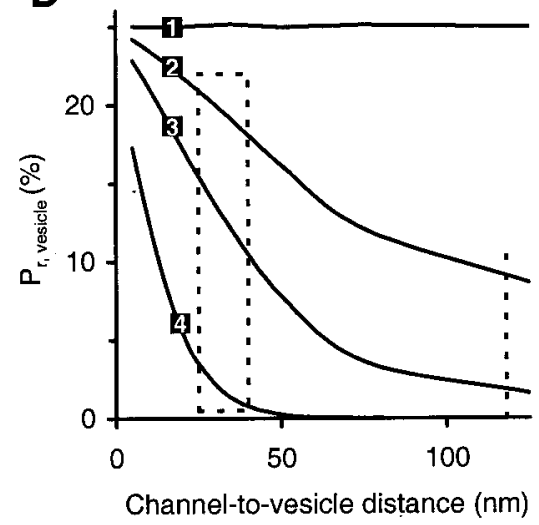

Figure 2. Effect of channel-to-vesicle distance on release probability. $A$, Reaction volume used in the simulation; all numbers are given in nanometers (not drawn to scale). Height $(400 \mathrm{~nm})$ is equal to the thickness of the calyx, and the width corresponds approximately to the distance between neighboring AZs (see Table 1). Partial 5 and $20 \mathrm{~nm}$ grids indicate varying spatial resolution (see Materials and Methods). Single $\mathrm{Ca}^{2+}$ channel is located at the center in the first voxel layer on the membrane (same for all channel-to-vesicle distances). Readily releasable vesicle is located on the membrane at $80 \mathrm{~nm}$ from the channel (example only). $B$, Peak $\mathrm{Ca}^{2+}$ current per channel required to yield release probability $\left(P_{\mathrm{r}, \text { vesicle }}\right)$ of $25 \%$ for the control condition [control $=$ endogenous fixed buffer (EFB) and ATP only] if all vesicles were located at the same distance from the $\mathrm{Ca}^{2+}$ channel that controls their release. $C$, Predicted peak $\left[\mathrm{Ca}^{2+}\right]$ at the location of the vesicle for the same assumption as in $B$. Traces 1-4 represent four different buffer conditions. Trace 1, EFB and ATP (control condition). Trace 2, EFB, ATP, and $10 \mathrm{~mm}$ EGTA-2. Trace 3, EFB, ATP, and 10 mm EGTA. Trace 4, EFB, ATP, and 1 mM BAPTA. $D$, Predicted $P_{\mathrm{r} \text {, vesicle }}$ for the same assumption as in $B$. Traces 1-4 for the buffer conditions are the same as in $C$. The dashed box indicates the distance range in which $P_{\mathrm{r} \text {, vesicle }}$ in the presence of EGTA, but not BAPTA, is similar to the experiment. Vertical dashed line indicates average vesicle distance $(118 \mathrm{~nm})$ of the reference topography used in the second part of Results. relative to $\left[\mathrm{Ca}^{2+}\right]_{\text {vesicle }}$ under control conditions is stronger the farther away from the channel the vesicle is located.

Figure $2 D$ shows $P_{\mathrm{r} \text {, vesicle }}$ as a function of the distance from the $\mathrm{Ca}^{2+}$ channel. As expected, for distances of $10 \mathrm{~nm}$ or less the efficacy of EGTA in reducing release is marginal. By comparing the differential effect of added exogenous buffers EGTA and BAPTA at any one distance, Figure $2 D$ confirms a previous result for the calyx (Naraghi and Neher, 1997). There is no distance at which the predicted $P_{\mathrm{r} \text {, vesicle }}$ is consistent with experimental results for both EGTA and BAPTA loading (assuming either parameter set, EGTA or EGTA-2). For any distance at which the predicted reduction of $P_{\mathrm{r} \text {, vesicle }}$ by $10 \mathrm{~mm}$ EGTA (Fig. 2D, trace 3) is similar to that observed in the experiments $(25-40 \mathrm{~nm})$ (Fig. $2 D$, dashed box), the predicted reduction of $1 \mathrm{mM}$ BAPTA is much stronger than that observed in the experiments. At distances $>125 \mathrm{~nm}$ the predicted reduction of $P_{\mathrm{r} \text {, vesicle }}$ of neither BAPTA nor EGTA is consistent with the experiments. (Fig. 2 shows simulations with Release Model A. Analogous simulations with Release Model B yielded the same result.)

In comparing the $P_{\mathrm{r} \text {, vesicle }}$ in Figure $2 D$ with experiments (which measure the average release probability across all vesicles in the calyx) we assumed implicitly that all readily releasable vesicles in the calyx are located at the same distance from their release-controlling channel. Under this assumption the experimental observation could not be explained. We therefore propose that the distance of individual vesicles to their release-controlling $\mathrm{Ca}^{2+}$ channel(s) varies across different release sites. This explanation indeed is suggested by the experiments. Although some vesicles seem to be located sufficiently far from $\mathrm{Ca}^{2+}$ channels to be affected by the slowly binding buffer EGTA $(10 \mathrm{~mm})$, other vesicles in the same calyx may be located closer to channels so that even the fast binding buffer BAPTA $(1 \mathrm{~mm})$ reduces their release probability only moderately.

To test this assumption, we assumed a simple distribution of distances. Every AZ is a circular area with the same radius of 125 $\mathrm{nm}$ (see Table 1; for the simple distribution the variation of AZ sizes was neglected). Every AZ has only a single $\mathrm{Ca}^{2+}$ channel controlling the release. The channel is located at the center of the AZ. Vesicles are located at random anywhere on the AZs, with a distance ranging between 0 and $125 \mathrm{~nm}$ from the center, the average distance being $83 \mathrm{~nm}$ (Fig. $3 A$ ). The predicted average $P_{\mathrm{r} \text {, vesicle }}$ across all vesicles is $25.0 \%$ for the control condition, $15.4 \%$ when adding $10 \mathrm{~mm}$ EGTA, and $7.7 \%$ when adding $1 \mathrm{~mm}$ BAPTA $\left(i_{\text {Ca, peak }}=0.53\right.$ pA per channel, Release Model A). Even for this simple distribution the predicted average $P_{\mathrm{r} \text {, vesicle }}$ is similar to the $P_{\mathrm{r}}$ observed in experiments with added buffers (Fig. $3 B)$. The quantitative mechanism that favors variable location of vesicles in reproducing the experimental data will be illustrated further below.

\section{Property II: Release-relevant $\mathrm{Ca}^{2+}$ sources are separated by diffusion distances $>200 \mathrm{~nm}$}

During APs at the calyx many $\mathrm{Ca}^{2+}$ channels open for every vesicle that is released (Borst and Sakmann, 1996). The experimental findings on the effects of added exogenous buffers on $P_{\mathrm{r}}$ may be used to infer a minimum distance between the $\mathrm{Ca}^{2+}$ channels that control phasic release. For these simulations the channels were arranged on a regular grid covering the entire presynaptic membrane (henceforth "periodic grid topography”) (Fig. $4 A$ ). The separation of neighboring channels (grid constant $d, 60 \mathrm{~nm}$ in Fig. $4 A, B$ ) was varied, adjusting the single channel conductance such that the predicted release probability for the 
A

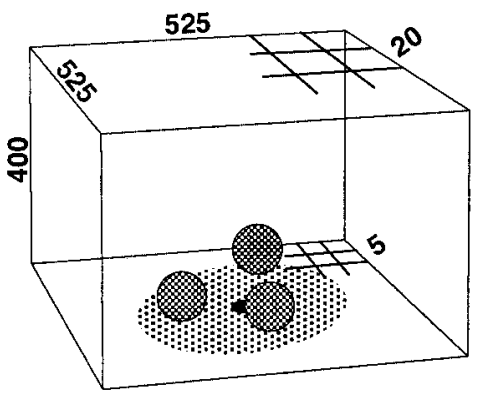

B

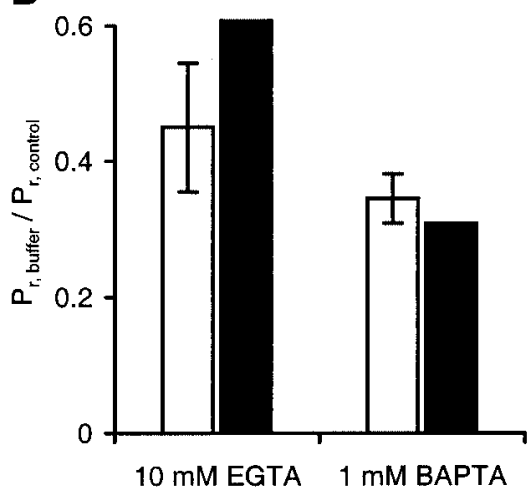

Figure 3. Measured buffer efficacies are reproduced (within \pm 2 SEM) when assuming variable channel-to-vesicle distance. $A$, Reaction volume used in the simulation; all numbers are given in nanometers (not drawn to scale). Partial 5 and $20 \mathrm{~nm}$ grids indicate varying spatial resolution (see Materials and Methods). Single $\mathrm{Ca}^{2+}$ channel is located at the center in the first voxel layer on the membrane. Readily releasable vesicles (three examples shown) are located randomly anywhere on the AZ (all AZs are a circular area with a $125 \mathrm{~nm}$ radius centered on the $\mathrm{Ca}^{2+}$ channel). $B$, Comparison of model-predicted $P_{\mathrm{r}}$ with measured $P_{\mathrm{r}}$. Filled columns, Predicted average $P_{\mathrm{r} \text {, vesicle }}$ for added exogenous buffer as ratio of control [control $=$ endogenous fixed buffer (EFB) and ATP only]. Open columns, Measured data for calyx. Error bars indicate \pm 1 SEM (see Table 2).

control condition was $25 \%$. Simulations used the uniform $i_{\mathrm{Ca}}$ mode.

Using $d=60 \mathrm{~nm}$ as an example, Figure $4 B$ illustrates how the $\mathrm{Ca}^{2+}$ domains generated by individual channels in a regular grid combine into a net $\left[\mathrm{Ca}^{2+}\right]$ transient (left axis), how this transient is modified in the presence of exogenous buffers, and how this affects the predicted release probability of vesicles at different hypothetical locations in the grid (right axis). For $d=60 \mathrm{~nm}$, there is no location at which $P_{\mathrm{r} \text {, vesicle }}$ in the presence of $1 \mathrm{~mm}$ BAPTA is similar to that measured in the experiments (i.e., 0.35 of $P_{\mathrm{r} \text {, vesicle }}$ under control conditions). In the immediate vicinity of a channel the peak of the $\left[\mathrm{Ca}^{2+}\right]$ transient in the presence of 1 mM BAPTA is approximately one-half of control. At this location, however, $P_{\mathrm{r} \text {, vesicle }}$ is only 0.05 of that under control conditions. For all other locations the release-reducing effect of $1 \mathrm{~mm}$ BAPTA relative to control is even stronger. This means that there can be no distribution of vesicles within the $d=60 \mathrm{~nm}$ grid that would be in agreement with the experimentally observed $P_{\mathrm{r}}$ (vesicles at fixed distance to the next channel, random locations, or any other distribution).

Extending the analysis, we varied $d$ between $5 \mathrm{~nm}$ (uniform influx through the membrane) and $500 \mathrm{~nm}$, thus varying the diffusion distances for $\mathrm{Ca}^{2+}$ between different $\mathrm{Ca}^{2+}$ channels. Keeping the total $\mathrm{Ca}^{2+}$ influx into the model calyx constant, we varied the single channel conductance as $d^{2}$. This leaves the peak of the average $\left[\mathrm{Ca}^{2+}\right]$ transient (average across all locations in grid) nearly constant (8-9 $\mu \mathrm{M}$ for control, 4.5-4.9 $\mu \mathrm{M}$ for $10 \mathrm{~mm}$ EGTA, 1.9-2.2 $\mu \mathrm{M}$ for $1 \mathrm{~mm}$ BAPTA). However, the spatial profile of the $\left[\mathrm{Ca}^{2+}\right]$ transients becomes increasingly nonuniform, leading to a steeper gradient between the spatial peaks of $\left[\mathrm{Ca}^{2+}\right]$ in the direct vicinity of channels and the troughs of $\left[\mathrm{Ca}^{2+}\right]$ between neighboring channels. For $d=480 \mathrm{~nm}$, the peak of the predicted $\left[\mathrm{Ca}^{2+}\right]$ transient $\left(\left[\mathrm{Ca}^{2+}\right]_{\text {peak }}\right)$ in the direct vicinity of channels is $350 \mu \mathrm{M}$, the peak of the average transient is $9.0 \mu \mathrm{M}$, and the peak at distance $d / 2$ from two neighboring channels is $4.1 \mu \mathrm{M}$ (Fig. $4 C$ ).

Similar to the analysis for Property I, we begin by investigating whether there is any hypothetical location in the grid of channels (i.e., all vesicles located at the same distance from nearest channel) for which the predicted $P_{\mathrm{r} \text {, vesicle }}$ is in agreement with the experiments with added exogenous buffers. At $d=5 \mathrm{~nm}$, $\left[\mathrm{Ca}^{2+}\right]_{\text {peak }}$ in the presence of $1 \mathrm{~mm}$ BAPTA is 0.24 of that of control. $P_{\mathrm{r} \text {, vesicle }}$ is only 0.0024 of control, i.e., $>100$ times smaller than that observed in the experiments (implying a supralinearity of $n=4.2$ when expressing $\left.P_{\mathrm{r} \text {, vesicle }} \propto\left[\mathrm{Ca}^{2+}\right]_{\text {peak }}^{n}\right)$. As seen in Figure $4, B$ and $C$, the reduction of $\left[\mathrm{Ca}^{2+}\right]_{\text {peak }}$ in the presence of exogenous buffers relative to $\left[\mathrm{Ca}^{2+}\right]_{\text {peak }}$ under control conditions is weaker the closer the location to any one channel and the larger the $d$. For example, if vesicles and channels were colocalized (in a grid of $d=200 \mathrm{~nm})$, then $\left[\mathrm{Ca}^{2+}\right]_{\text {peak }}$ in the presence of $1 \mathrm{~mm}$ BAPTA would be 0.75 of $\left[\mathrm{Ca}^{2+}\right]_{\text {peak }}$ under control conditions, and $P_{\mathrm{r} \text {, vesicle }}$ in the presence of $1 \mathrm{mM}$ BAPTA would be $\sim 0.75^{4}=$ 0.32 of $P_{\mathrm{r} \text {, vesicle }}$ under control conditions (in agreement with the experiments). For the same assumption, however, predicted $P_{\mathrm{r}, \text { vesicle }}$ in the presence of $10 \mathrm{~mm}$ EGTA would be too high (0.7 of control). Summarizing, there is no location within a grid of $\mathrm{Ca}^{2+}$ channels (for any $d$ ) at which the predicted effects of BAPTA and EGTA are both in agreement with the experiments.

Similar to the simulations for Property I, the agreement between measured and modeled $P_{\mathrm{r}}$ improves when a distribution of different locations of vesicles within the grid is considered. As a simple distribution of vesicles we assumed that vesicles are located at random anywhere within the grid of channels (exact dimensions of AZs, for the moment, were neglected). Then the predicted release probability of the calyx $\left(P_{\mathrm{r}, \text { calyx }}\right)$ is given by the average $P_{\mathrm{r} \text {, vesicle }}$ across all locations in the grid. Given such a simple distribution, $P_{\mathrm{r} \text {, calyx }}$ in the control condition is nearly constant for all $d$. However, the predicted efficacy of added exogenous buffers in reducing $P_{\mathrm{r} \text {, calyx }}$ changes by more than two orders of magnitude (Fig. 4D). When compared with experimental data, the results for EGTA (both parameter sets) as well as for BAPTA show that $d$ is likely to be at least $200 \mathrm{~nm}$. Otherwise, the expected effect of either exogenous buffer would be far stronger than that observed in the experiments. To eliminate the uncertainty introduced by the role of ATP (for which the kinetic parameters and concentration in the native calyx are uncertain), we repeated the simulations without ATP and simulated the effect of BAPTA and endogenous fixed buffer alone $(50 \mu \mathrm{M}$ vs $1 \mathrm{~mm}$ BAPTA; see Materials and Methods for effect of endogenous fixed buffer). The results also imply $d>200 \mathrm{~nm}$. (Fig. 4 shows simulations with Release Model A. Analogous simulations with Release Model B yielded similar results, suggesting $d>250 \mathrm{~nm}$.)

In the simulations assuming a periodic grid of $\mathrm{Ca}^{2+}$ channels, we have, so far, used the uniform $i_{\mathrm{Ca}}$ mode (i.e., every channel of the grid opens during an AP). However, because only $10-20 \%$ of 
A

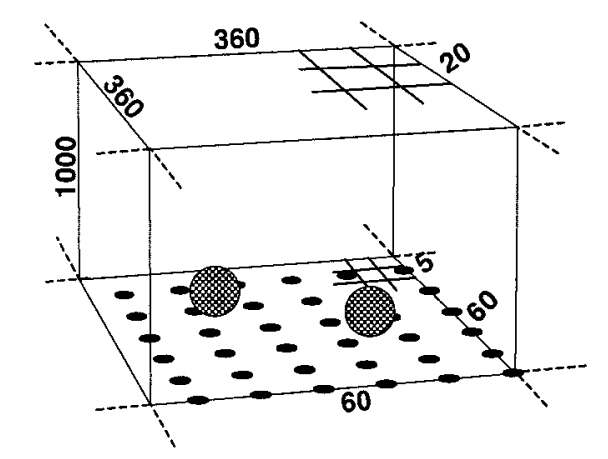

B

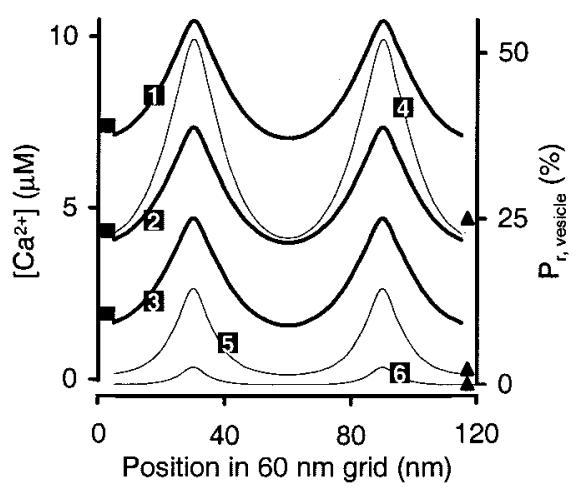

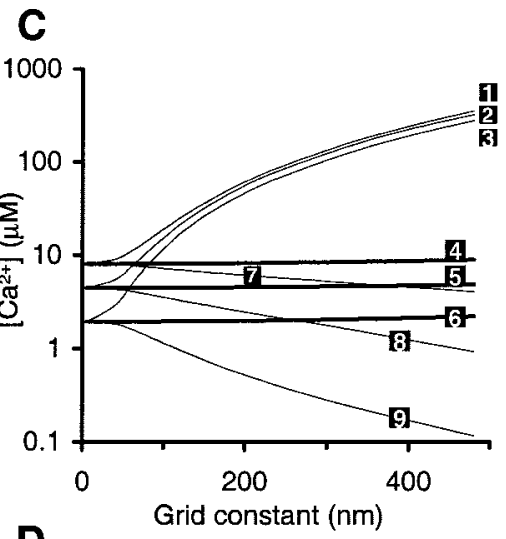

D

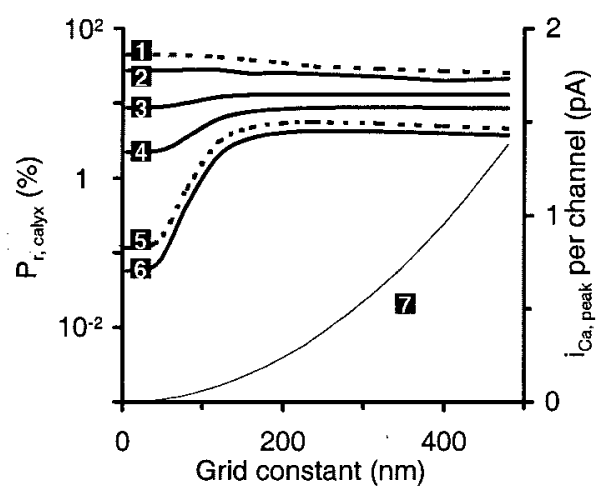

Figure 4. Effect of $\mathrm{Ca}^{2+}$ channel spacing on release probability. $A$, Reaction volume with the periodic grid topography used in the simulation; all numbers are given in nanometers (not drawn to scale). Partial 5 and $20 \mathrm{~nm}$ grids indicate varying spatial resolution. Dashed lines indicate repetition of volume with the use of periodic boundary conditions (see Materials and Methods). $\mathrm{Ca}^{2+}$ channels are located on a uniform grid with grid constant $d$ (example shows $d=60 \mathrm{~nm}$ ). Readily releasable vesicles are located randomly anywhere on the membrane. $B$, Spatial profiles of $\left[\mathrm{Ca}^{2+}\right]$ (traces 1-3, snapshot at time of peak $I_{\mathrm{Ca}}$ ) and of $P_{\mathrm{r} \text {, vesicle }}$ (traces 4-6, after 5 msec; right axis) generated by a $d=60 \mathrm{~nm}$ regular grid of channels under different buffer conditions; single $\mathrm{Ca}^{2+}$ channel current the same as in $D$. Channels are at 30 and $90 \mathrm{~nm}$. Traces 1, 4, Endogenous fixed buffer (EFB) and ATP (control condition). Traces 2, 5, EFB, ATP, and 10 mM EGTA. Traces 3, 6, EFB, ATP, and $1 \mathrm{~mm}$ BAPTA. Squares at left axis indicate average $\left[\mathrm{Ca}^{2+}\right]$ across the membrane (7.4, 4.3, and 1.9 $\mu \mathrm{M}$ for control, EGTA, and BAPTA, respectively). Triangles at right axis indicate average $P_{\mathrm{r}, \text { vesicle }}\left(=P_{\mathrm{r}, \text { calyx }}\right)$ across the membrane (25, 2.4 , and $0.13 \%$ for control, EGTA, and BAPTA, respectively). $C$, Peaks of $\left[\mathrm{Ca}^{2+}\right]$ transients for different grid constants and buffer conditions $(1,4$, and $7 ; 2,5$, and $8 ; 3,6$, and 9 as in $B$; single $\mathrm{Ca}^{2+}$ channel current same as in $D$ ). 1-3, Peak of transient at $10 \mathrm{~nm}$ above a channel. 4-6, Peak of average transient across the membrane. 7-9, Peak of tran-

sient at half-grid constant from two neighboring channels. $D$, Predicted average $P_{\mathrm{r} \text {, vesicle }}$ across the membrane $\left(=P_{\mathrm{r} \text {, calyx }}\right)$ as a function of grid constant $d$. Traces 1- 6 show $P_{\mathrm{r} \text {, calyx }}$ for six different buffer conditions. Trace 1, EFB and $50 \mu \mathrm{M}$ BAPTA. Trace 2, EFB and ATP (control condition). Trace 3, EFB, ATP, and 10 mm EGTA-2. Trace 4, EFB, ATP, and 10 mm EGTA. Trace 5, EFB and 1 mm BAPTA. Trace 6, EFB, ATP, and 1 mm BAPTA. Trace 7 , Peak $i_{\mathrm{Ca}}$ per channel required to achieve $P_{\mathrm{r}, \text { calyx }} \sim 25 \%$ for the control condition (right axis).

all $\mathrm{Ca}^{2+}$ channels may open during a single AP (Colecraft et al., 2001), the topographic pattern of open $\mathrm{Ca}^{2+}$ channels is different from the pattern of all, i.e., open or closed channels. In particular, the average diffusion distance of $\mathrm{Ca}^{2+}$ between open channels may be large $(>200 \mathrm{~nm})$, even if these open channels are part of a periodic channel grid with $d<200 \mathrm{~nm}$.

To illustrate this, we might consider, for example, the following situation. Each vesicle is surrounded by a large uniform grid of $\mathrm{Ca}^{2+}$ channels (Yamada and Zucker, 1992). We used a grid of $10 \times 10$ channels, with a grid constant of $50 \mathrm{~nm}$. The readily releasable vesicle was located in the middle of the channel field, in our example colocalized with one of the $\mathrm{Ca}^{2+}$ channels ("nonperiodic grid topography”) (Fig. 5A). Time course and amplitude of the $\mathrm{Ca}^{2+}$ currents were varied across channels (stochastic $i_{\mathrm{Ca}}$ mode; see Materials and Methods). To simulate low open probability of $\mathrm{Ca}^{2+}$ channels during APs, we added a third gate to the two-gate Hodgkin-Huxley Model. The third gate, for which gating was independent of the membrane potential, either opened for the entire course of an AP (probability $p_{\text {open, max }}$ ) or remained closed. Thus, the peak open probability of a single $\mathrm{Ca}^{2+}$ channel during an AP was $p_{\text {open }}=69 \% \cdot p_{\text {open, max }}$, where $69 \%$ is the predicted peak open probability of the two-gate Hodgkin-Huxley Model for the AP waveform (see Materials and Methods) and $p_{\text {open, max }}$ was varied between 100 and $15 \%$. To yield $P_{\mathrm{r} \text {, calyx }}$ $\sim 25 \%$ for the control condition, we set single channel conductance to $0.40 \mathrm{pS}$ for $p_{\text {open }}=69 \%$ and scaled it as $1 / p_{\text {open }}$ up to 2.67 $\mathrm{pS}$ for $p_{\text {open }}=10.4 \%$ (Release Model A). This left the average total $\mathrm{Ca}^{2+}$ influx per AP unchanged, independent of $p_{\text {open }}$ $\left(i_{\text {Ca, peak }}\right.$ was $0.018 \mathrm{pA}$ per channel, averaged across all open and closed channels).

Figure $5 B$ shows predicted effects of added 1 mm BAPTA on $P_{\text {r, calyx }}$. The results demonstrate how the effective topography (open $\mathrm{Ca}^{2+}$ channels relative to vesicle) changes with decreasing $p_{\text {open }}$ and how this affects whether the topography reproduces the experimental data. For $p_{\text {open }}=69 \%$, the predicted releasesuppressing effect of added $1 \mathrm{~mm}$ BAPTA is much stronger than that observed in the experiments (as expected from the result for $d=50 \mathrm{~nm}$ in Fig. $4 D$ ). $P_{\mathrm{r} \text {, calyx }}$ with added $1 \mathrm{~mm}$ BAPTA is $0.04 \pm$ 0.005 of $P_{\mathrm{r}, \text { calyx }}$ under control conditions, i.e., approximately eight times lower than measured in the experiments (all values given as mean \pm SEM after 200-800 Monte Carlo simulations). This is expected, because for high $p_{\text {open }}$ the diff usional distances between open $\mathrm{Ca}^{2+}$ channels are too small. In contrast, for $p_{\text {open }}=10 \%$, most open $\mathrm{Ca}^{2+}$ channels are separated by diffusion distances $>200 \mathrm{~nm}$. Thus the predicted effect of $1 \mathrm{~mm}$ BAPTA is consistent with experimental data $\left(P_{\mathrm{r} \text {, calyx }}\right.$ reduced to $0.26 \pm 0.06$ of control). (As indicated in Table 3 , the nonperiodic grid topography, although it is consistent with experiments with added BAPTA, is not consistent with experiments measuring the apparent Hill coefficient $m$; see Property III.)

For diffusion distances $d$ of several hundred nanometers, required $i_{\mathrm{Ca} \text {, peak }}$ per channel is on the order of $1 \mathrm{pA}$ (Fig. 4D, right axis). This current is $\sim 5-10$ times higher than the usual upper estimates for single $\mathrm{Ca}^{2+}$ channels (conductance $\sim 2 \mathrm{pS}$ at physiological conditions; Gollasch et al., 1992; Church and Stanley, 1996). For large $d$, therefore, we replaced each single channel 

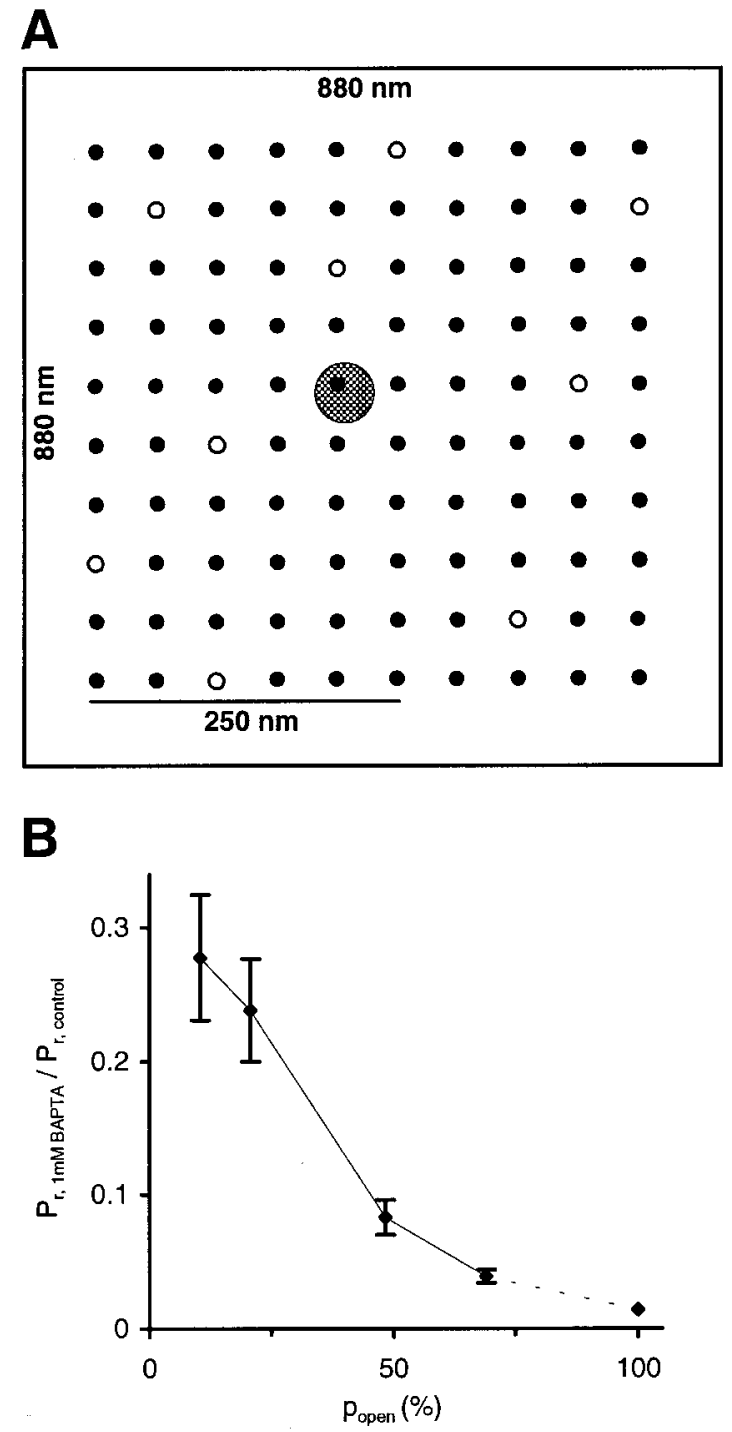

Figure 5. Effect of $\mathrm{Ca}^{2+}$ channel open probability on release probability. $A$, Reaction volume with the nonperiodic grid topography (100 channels on $d=50 \mathrm{~nm}$ grid) used in the simulation (height of reaction volume, 500 $\mathrm{nm})$. Readily releasable vesicle is colocalized with the $\mathrm{Ca}^{2+}$ channel at the center of the grid. $B$, Predicted $P_{\mathrm{r} \text {, vesicle }}$ after adding $1 \mathrm{~mm}$ BAPTA as a ratio of $P_{\mathrm{r}, \text { vesicle }}$ under the control condition (control $=$ endogenous fixed buffer and ATP only) as a function of the open probability of $\mathrm{Ca}^{2+}$ channels $\left(p_{\text {open }}\right)$ assumed in the three-gate channel model. For decreasing $p_{\text {open }}$, single channel conductance was increased as $1 / p_{\text {open }}$ (see Property II for values). Results for ratios show mean \pm SEM after 200-1000 Monte Carlo simulations for each data point (stochastic $i_{\mathrm{Ca}}$ mode). The ratio for $p_{\text {open }}=100 \%$ is the one predicted by the uniform $i_{\mathrm{Ca}}$ mode (i.e., all channels open). Control condition: $P_{\mathrm{r} \text {, vesicle }}=30 \% \pm 1.5 \%$ for $p_{\mathrm{open}}=$ $10.4 \% ; P_{\mathrm{r} \text {, vesicle }}=35 \% \pm 1.5 \%$ for $p_{\text {open }}=69 \% ; P_{\mathrm{r} \text {, vesicle }}=35.2 \%$ for $p_{\text {open }}=100 \%$.

with a cluster of 10 channels, each conducting only one-tenth of the original channel (channel-to-channel distance within cluster, $\sim 15 \mathrm{~nm}$ ). Predicted effects of added exogenous buffers remained almost unchanged (data not shown). The $\left[\mathrm{Ca}^{2+}\right]$ transient provided by a single large channel is, for many quantitative arguments, indistinguishable from that of a cluster of channels. Henceforth, we will refer to either as a $\mathrm{Ca}^{2+}$ source.

In summary, we conclude that, for any hypothesized location of vesicles within a large field of channels or channel clusters, the average distance between neighboring (open) $\mathrm{Ca}^{2+}$ sources is likely to be $>200 \mathrm{~nm}$.

\section{Buffer effects in single channel versus multiple channel topographies}

Our result, at a first glance, may seem to contradict previous interpretations of experiments with added exogenous buffers. Previously, a substantial effect of the kinetically slow buffer EGTA in reducing $P_{\mathrm{r}}$, particularly when compared with the effects of kinetically fast BAPTA, was used to infer relatively large diffusion distances for $\mathrm{Ca}^{2+}$ between release-controlling channels and vesicles (Borst and Sakmann, 1996). This is true when assuming that a vesicle is triggered by a single $\mathrm{Ca}^{2+}$ channel/source. The larger the distance from a single $\mathrm{Ca}^{2+}$ source, the stronger the predicted relative reduction of phasic release by mobile buffers (Neher, 1998b) (Fig. 2D). However, for the case of a grid of channels, the effect is more complicated. The larger the grid distance $d$, i.e., the larger the average diff usion distance between vesicles and their nearest channels, the less efficient the buffers are in reducing phasic release. This can be understood by approximating $\left[\mathrm{Ca}^{2+}\right]_{\text {vesicle }}$ by adding up, for any one location in the grid, the single domains of all other channels (linearized steady-state approximation; Neher, 1998b). The smaller the $d$, the more spatially uniform are the $\left[\mathrm{Ca}^{2+}\right]$ transients (along the membrane) and thus the more generated not only by a single nearby channel but by a large number of channels at different locations (Fig. $4 C$ ). In addition, because $P_{\mathrm{r} \text {, vesicle }}$ is a nonlinear function of $\left[\mathrm{Ca}^{2+}\right]_{\text {vesicle }}$, it is not sufficient to consider the effect of added exogenous buffers on $\left[\mathrm{Ca}^{2+}\right]_{\text {vesicle }}$ alone. Instead, when considering distributions of vesicle distances, the effect of the buffers on the average release probability must be considered, too. (As seen in Fig. 4, the effect of the buffers on the average $\left[\mathrm{Ca}^{2+}\right]_{\text {vesicle }}$ is independent of $d$, whereas the effect on average $P_{\mathrm{r} \text {, vesicle }}$ changes by more than two orders of magnitude.)

The above finding relates primarily to the probable location of $\mathrm{Ca}^{2+}$ sources with respect to other $\mathrm{Ca}^{2+}$ sources, not to the location of vesicles. As shown, inferring diffusion distances between channels and vesicles from exogenous buffer experiments depends on previous assumptions on how many $\mathrm{Ca}^{2+}$ channels contribute to the local $\left[\mathrm{Ca}^{2+}\right]$ at the vesicle (single channel domain vs multiple channels domain). Therefore, we will address the question of how many $\mathrm{Ca}^{2+}$ channels control the release of a vesicle in a separate, independent argument.

Property III: The majority of vesicles is controlled by clusters of $\sim 10$ or more $\mathrm{Ca}^{2+}$ channels

Experimental modifications of the stochastic gating of $\mathrm{Ca}^{2+}$ channels and/or their partial blockage by toxins affect phasic transmitter release at the calyx (see Table 2). Such experiments have been used to conclude that the majority of vesicles at this synapse is controlled by more than one channel (Borst and Sakmann, 1999a; Wu et al., 1999). Extending these results, we estimate how many $\mathrm{Ca}^{2+}$ channels are likely to control the release of a single vesicle and how these channels are located.

Time-independent model without diffusion. We begin with a simple model (Yoshikami et al., 1989), which assumes the following: (1) The release probability $\left(P_{\mathrm{r} \text {, vesicle }}\right)$ of every vesicle is proportional to the $n$th power of $\left[\mathrm{Ca}^{2+}\right]_{\text {vesicle }}, P_{\mathrm{r} \text {, vesicle }} \propto$ $\left[\mathrm{Ca}^{2+}\right]_{\text {vesicle }}^{n}\left[\mathrm{Ca}^{2+}\right]_{\text {vesicle }}$ is supplied by one or more channels controlling the release of the vesicle. (2) The total number of channels controlling each vesicle $(N)$, open or closed, is the same at every release site. (3) During an AP a channel either opens 
A

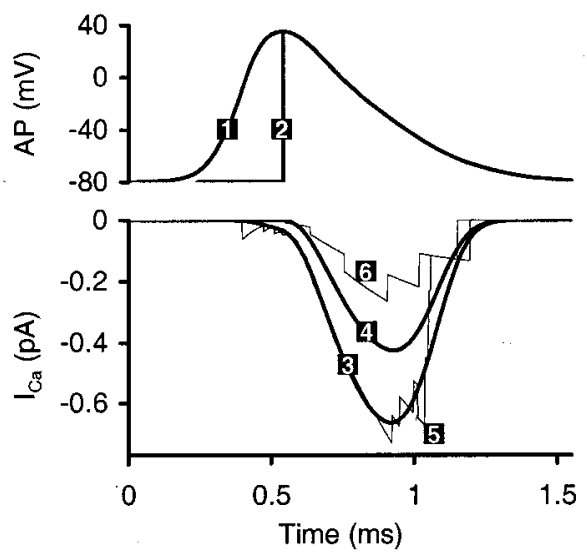

B

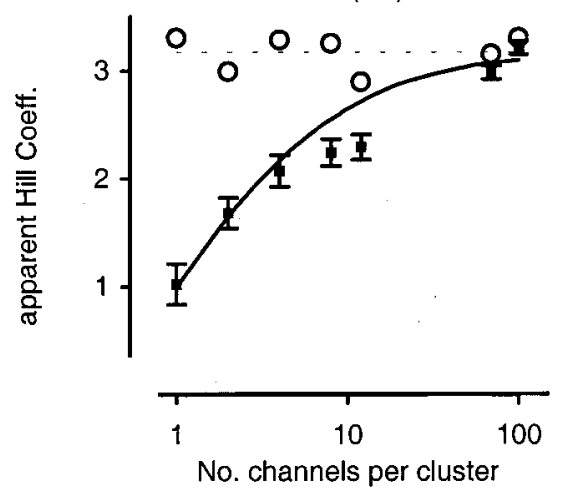

C
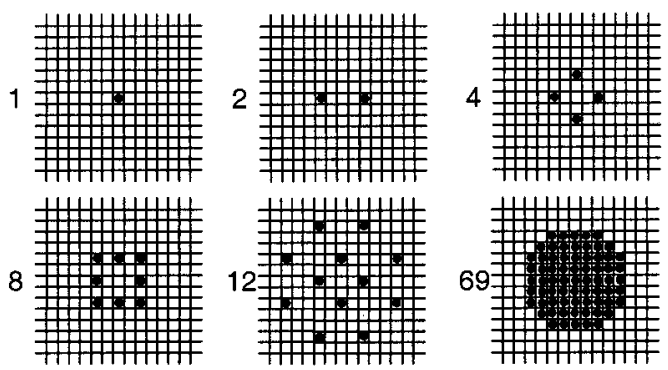

D

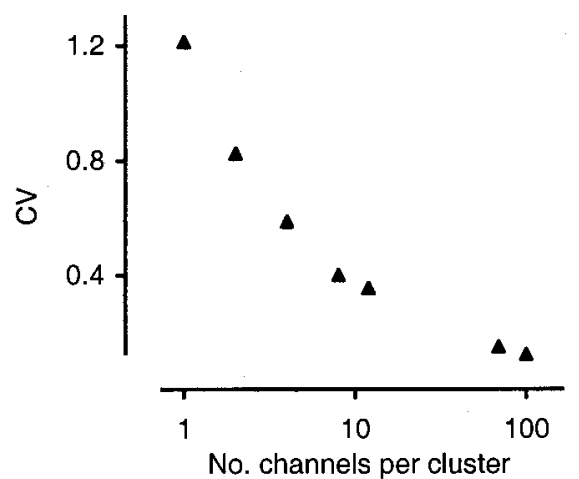

Figure 6. Effect of the number of $\mathrm{Ca}^{2+}$ channels on release probability. $A, I_{\text {Ca }}$ predicted by Hodgkin-Huxley Model for different AP waveforms. Trace 1, Physiological AP. Trace 2, Step AP to reduce peak channel open probability $\left(p_{\text {open }}\right)$. Trace 3 , Average $I_{\mathrm{Ca}}$ (used in uniform $i_{\mathrm{Ca}}$ mode) for 12 channel cluster (physiological AP); half-width of $I_{\mathrm{Ca}}=383 \mu \mathrm{sec}$; $p_{\text {open }}=69 \%$. Trace 4, Same as trace 3 but for step AP; $p_{\text {open }}=42 \%$. Trace 5 , Example of stochastic $I_{\mathrm{Ca}}$ (used in stochastic $i_{\mathrm{Ca}}$ mode) for cluster of 12 channels (physiological AP). Trace 6, Same as trace 5 but for step AP. B, Effects of the number of channels per cluster on apparent Hill coefficient $m$ (see Property III for details). Solid line, $m$ predicted by timeindependent model. Squares, $m$ predicted by time-dependent model with the stochastic $i_{\mathrm{Ca}}$ mode (for all $N$ the vesicle locations are as described for the reference topography). Error bars indicate \pm SEM after 400 (probability $p_{\text {open }}$ ) or remains closed (no multiple opening). (4) Each open channel contributes the same $\left[\mathrm{Ca}^{2+}\right]$ to $\left[\mathrm{Ca}^{2+}\right]_{\text {vesicle. }}$. Assuming that sites are independent, we get: $P_{\mathrm{r} \text {, calyx }}=$ $\left(P_{\mathrm{r} \text {, vesicle }}\right)_{\text {average }} \propto \Sigma_{\mathrm{k}=1}{ }^{N} p(k) \cdot k^{n}$, where $p(k)$ is the probability that, at any single site, $k$ of the $N$ channels open during an AP (binomial distribution). Because channel gating is stochastic, the number of open channels at each release site varies around the average number $\left(p_{\text {open }} \cdot N\right)$. This causes a variance of $\left[\mathrm{Ca}^{2+}\right]_{\text {vesicle }}$ across sites. Therefore, only the average $\left[\mathrm{Ca}^{2+}\right]_{\text {vesicle }}$ of all release sites is reduced proportionally to $p$. If $n \neq 1$, the effect on release is nonlinear, i.e., the average release probability of all vesicles in the calyx $\left(P_{\mathrm{r} \text {, calyx }}\right)$ is not proportional to the average $\left[\mathrm{Ca}^{2+}\right]_{\text {vesicle }}$ raised to the $n$th power. Instead, $P_{\mathrm{r}, \text { calyx }} \propto p_{\text {open }}{ }^{m}$ [the notation of $m$ vs $n$ follows the one in Wu et al. (1999), defined in Table 2]. Therefore, the apparent degree of supralinearity of release versus $\mathrm{Ca}^{2+}$ influx measured experimentally $(m)$ may be different from $n$ and depends on how the influx is varied (Yoshikami et al., 1989; Quastel et al., 1992). The discrepancy of $m$ versus $n$ is larger the higher the coefficient of variation $(\mathrm{CV})$ of $\left[\mathrm{Ca}^{2+}\right]_{\text {vesicle }}$ across release sites. $\mathrm{CV}$, which depends on the number of $\mathrm{Ca}^{2+}$ channels per site, is largest for the case of a single channel, for $N=1, m=1$, independent of $n$ (Yoshikami et al., 1989; Augustine et al., 1991). As the number of channels controlling each site increases, the $\mathrm{CV}$ of $\left[\mathrm{Ca}^{2+}\right]_{\text {vesicle }}$ decreases and $m$ converges to $n$.

$\mathrm{N}$ estimated with time-independent model. In presynaptic voltage-clamp recordings at the calyx, Borst and Sakmann (1999a) reduced $p_{\text {open }}$ for $\mathrm{Ca}^{2+}$ channels by modifying the AP waveform. To simulate these experiments with the timeindependent model, we calculated $P_{\mathrm{r}, \text { calyx }}$ for $p_{\text {open }}=69 \%$ (for the physiological AP waveform) (Fig. 6A) and for $p_{\text {open }}=42 \%$ (step-like AP waveform) and determined $m$ according to $P_{\mathrm{r}, \text { calyx }}$ $\propto p_{\text {open }}{ }^{m}$ (both $p_{\text {open }}$ given by a two-gate Hodgkin-Huxley Model fit to the calyx as in Borst and Sakmann, 1998). We assumed $n=$ 3.3 (maximum possible $m$ predicted by time-dependent diffusion model; see below). As expected, $m$ predicted by the timeindependent model was equal to 1 for $N=1$ and converged to 3.3 for large $N$ (Fig. $6 B$, solid line). On the basis of the experimental finding that $m \sim n$, the predictions for $m$ suggest that phasic transmitter release for the majority of vesicles is likely to be controlled by at least $10 \mathrm{Ca}^{2+}$ channels per vesicle. Although in the model $m$ is a function of $p_{\text {open }}$ (high vs low), the inferred minimum number of channels $N$ is insensitive to the exact values for $p_{\text {open }}$. We also tested $p_{\text {open }}=75$ versus $25 \%($ yielding $N>11)$ and $p_{\text {open }}=20$ versus $10 \%$ (yielding $N>14$ ).

Time-dependent model with diffusion. The simplified model above neglects time-dependent buffered diff usion of $\mathrm{Ca}^{2+}$ as well as the exact time-dependent response of vesicles to transient

Monte Carlo simulations for each data point. Open circles, Apparent Hill coefficient predicted by time-dependent model with the uniform $i_{\mathrm{Ca}}$ mode. $C$, Number and position of $\mathrm{Ca}^{2+}$ channels in clusters of different $N$ $(1-100)$ used in the simulation. Grid indicates $(5 \mathrm{~nm})^{3}$ voxels on the membrane; the black circles indicate a voxel with a channel. The cluster with $N=100$ channels is the same as the cluster with $N=1$ channel except that the same voxel holds 100 channels instead of 1 (unrealistic channel density). Reaction volume, as well as location of readily releasable vesicles and of cluster centers (indicated by the black circle in $N=1$ cluster) on $\mathrm{AZs}$, is as in Figure $7 A$. $D$, Coefficient of variation of the total $\mathrm{Ca}^{2+}$ influx through a cluster [time integral of $I_{\mathrm{Ca}}(t), 0-5 \mathrm{msec}$ ] as a function of the number of channels per cluster (physiological AP waveform). 
$\left[\mathrm{Ca}^{2+}\right]$. To confirm the result on $N$, we implemented stochastic channel gating into the three-dimensional time-dependent model used to infer Properties I and II. Vesicles and clusters of $\mathrm{Ca}^{2+}$ channels were located randomly on $\mathrm{AZs}$ as described for the reference topography. Time course and amplitude of the $\mathrm{Ca}^{2+}$ currents were varied across channels (stochastic $i_{\mathrm{Ca}}$ mode; see Materials and Methods). Channel kinetics were driven either by the physiological AP waveform, resulting in $p_{\text {open }}=69 \%$, or by the modified step-like waveform $\left(p_{\text {open }}=42 \%\right)$. Total conductance per channel cluster was $4.8 \mathrm{pS}$ or $4.8 \mathrm{pS} / N$ per channel. [4.8 $\mathrm{pS}$ is 0.33 of the conductance used to simulate release under control conditions. The lower conductance, which simulates lower $\left[\mathrm{Ca}^{2+}\right]$ of the extracellular solution, was used so that $n_{\text {model }} \sim 3$ (average $n$ observed in the experiments; see Table 2).]

$\mathrm{N}$ estimated with time-dependent model. When channels are gated by the step-like AP, the total influx into the calyx is $61 \%$ of that under the physiological AP. The average release probability $P_{\text {r, calyx }}$ is reduced to $0.61^{m}$, where the value of $m$ is strongly dependent on $N$ (Fig. 6B). We show results for seven cluster types with $N$ varying between 1 and 100 channels per cluster (Fig. $6 C$ ). To confirm that the predicted change in $m$ is attributable to the change in the $\mathrm{CV}$ (Fig. $6 D$ ) of the total $\mathrm{Ca}^{2+}$ influx per cluster and not attributable to different channel-to-vesicle distances inherent in clusters of varying $N$, we repeated the simulations for all seven clusters. This time we did not vary individual channel currents stochastically but used the uniform $i_{\mathrm{Ca}}$ mode. As expected, the predicted Hill coefficient is $\sim 3.3$ for all $N$ (Fig. $6 B$, open circles).

The time-dependent model confirms the finding on $N$ derived from the time-independent model. However, for $N=4-12$, an additional effect is revealed. Because the $\mathrm{Ca}^{2+}$ channels in the cluster cannot all be in the same location in the membrane, the diffusion distance to the vesicle they control varies and thus the $\left[\mathrm{Ca}^{2+}\right]$ that each open channel contributes to the combined $\left[\mathrm{Ca}^{2+}\right]_{\text {vesicle }}$ varies as well. Therefore, variable diff usion distances of the channels controlling a particular vesicle raises the $\mathrm{CV}$ of $\left[\mathrm{Ca}^{2+}\right]_{\text {vesicle }}$ (for the same $N$ ), thus increasing the discrepancy between the measured values of $m$ versus $n$. To illustrate this further, we may consider again the nonperiodic grid topography simulated in Property II (Fig. $5 A$ ). At high $p_{\text {open }}=69 \%$, the prediction for $m(2.5 \pm 0.1)$ is consistent with the experiments, because at high $p_{\text {open }}$ each vesicle is controlled by a large number of $\mathrm{Ca}^{2+}$ channels at similar distances ( $m$ given as mean \pm SEM after 200-800 Monte Carlo simulations). At low $p_{\text {open }}=10 \%$, a vesicle still is controlled by $\sim 10 \mathrm{Ca}^{2+}$ channels (for the grid of 100 channels). However, in the grid these channels are not located at similar distances from the vesicle. Therefore, the predicted $m$ for $p_{\text {open }}=10 \%$ is only $1.37 \pm 0.14$, far lower than that measured in the experiments.

In summary, we conclude that $N \sim 10$ or more $\mathrm{Ca}^{2+}$ channels control the phasic release of a single vesicle at the majority of release sites at the calyx and, further, that these channels are located at similar distances from the vesicle they control. For the $N=12$ cluster in Figure $6 B$, the average vesicle, located at $118 \mathrm{~nm}$ from the cluster center, is located at $90-140 \mathrm{~nm}$ from an individual channel.

It should be noted that the above simulations are based on the assumption that the Hodgkin-Huxley Model is a sufficiently accurate description for gating and current of single $\mathrm{Ca}^{2+}$ channels. The model is probably not accurate for all $\mathrm{Ca}^{2+}$ channel subtypes. Just as variable distance between channels and vesicles increases the variance of $\left[\mathrm{Ca}^{2+}\right]_{\text {vesicle }}$ (see above), different sub- types of $\mathrm{Ca}^{2+}$ channels with presumably different gating and/or conductances would produce the same effect, thus further increasing the discrepancy between $m$ and $n$ (for the same $N$ ). Similarly, if $p_{\text {open }}$ during APs is lower than $69 \%$ assumed in the above simulations, more channels per cluster would be needed to achieve sufficiently low $\mathrm{CV}$ of the $\left[\mathrm{Ca}^{2+}\right]$ reaching the vesicles. Hence our estimate $N \sim 10$ should be viewed as a lower limit.

Property synthesis: Phasic release is controlled by one or a few channel clusters per AZ, and vesicles are located at variable distance from the cluster(s)

Properties I-III describe three specific "requirements" on the topography of release sites at the calyx. Although there are many conceivable topographies that would exhibit one or two of these properties, very few topographies exhibit all three properties simultaneously. Further combining these requirements with recent anatomical data of the location and size of AZs in the presynaptic membrane of the calyx, one can infer a single, probable topography for the calyx.

Electron microscopic (EM) reconstruction of the calyx shows that AZs are separated by $200-800 \mathrm{~nm}$ from the next closest AZ (see Table 1). The average radius of an AZ is $125 \mathrm{~nm}$. Therefore, $>200 \mathrm{~nm}$ separation of $\mathrm{Ca}^{2+}$ sources (Property II) suggests that phasic release for the majority of $\mathrm{AZs}$ is controlled by a single source of $\mathrm{Ca}^{2+}$ per AZ, a source being a single $\mathrm{Ca}^{2+}$ channel or a group of $\mathrm{Ca}^{2+}$ channels.

As for the distances between $\mathrm{Ca}^{2+}$ channels and vesicles, the simulations of the effects of added exogenous buffers (Property I) and of reduced $\mathrm{Ca}^{2+}$ channel open probability (Property III) suggest seemingly contradicting properties. Simulations for exogenous buffer suggest nonuniformity (variable distances), whereas simulations for reduced $p_{\text {open }}$ suggest uniformity (similar distances). However, these two properties are not mutually exclusive and may be treated as independent requirements on the topography. The nonuniformity relates to the distances that different releasable vesicles have to the $\mathrm{Ca}^{2+}$ source that controls the release of the vesicles. The uniformity relates to the distances that one vesicle has to the several individual channels of the $\mathrm{Ca}^{2+}$ source.

To satisfy both requirements in one topography, we suggest that $\mathrm{Ca}^{2+}$ channels at AZs appear in clusters, with 10 or more channels per cluster and with a maximum distance between any two channels in the cluster of $\sim 50 \mathrm{~nm}$. Ten $\mathrm{Ca}^{2+}$ channels on a circular area with a diameter of $50 \mathrm{~nm}$ correspond to one channel per $14 \times 14 \mathrm{~nm}^{2}$ membrane area. This is consistent with estimates of channel-to-channel distances for other synapses (Stanley, 1997). For any one vesicle located, for example, $100 \mathrm{~nm}$ away from the center of the cluster, the distances between the $\mathrm{Ca}^{2+}$ channels and this vesicle are "similar" (75-125 nm). As a second, independent property of the reference topography, we suggest that different releasable vesicles (at the same AZ or at other AZs) are located at different distances from the $\mathrm{Ca}^{2+}$ channel cluster. Note that the simulations to reproduce the measured effects of added exogenous buffers on $P_{\mathrm{r}}$ cannot predict the exact distribution of cluster-to-vesicle distances. However, the simulations indicate that the $\mathrm{CV}$ of the distribution must be $\sim 0.5$ or larger.

\section{Spatiotemporal pattern of $\left[\mathrm{Ca}^{2+}\right]$ transients and phasic transmitter release}

We have derived the topographic properties likely to be found at release sites of the calyx. However specific, these properties do not define the topography in every detail. In particular, they cannot define the exact distances between readily releasable ves- 
A

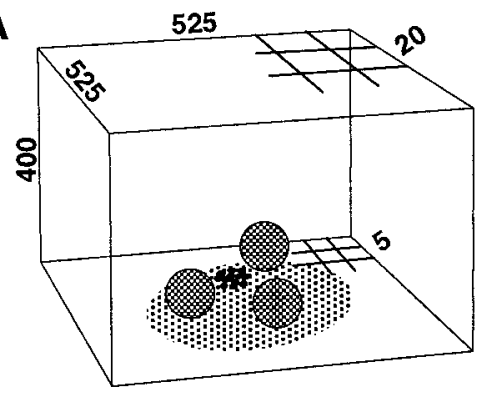

B
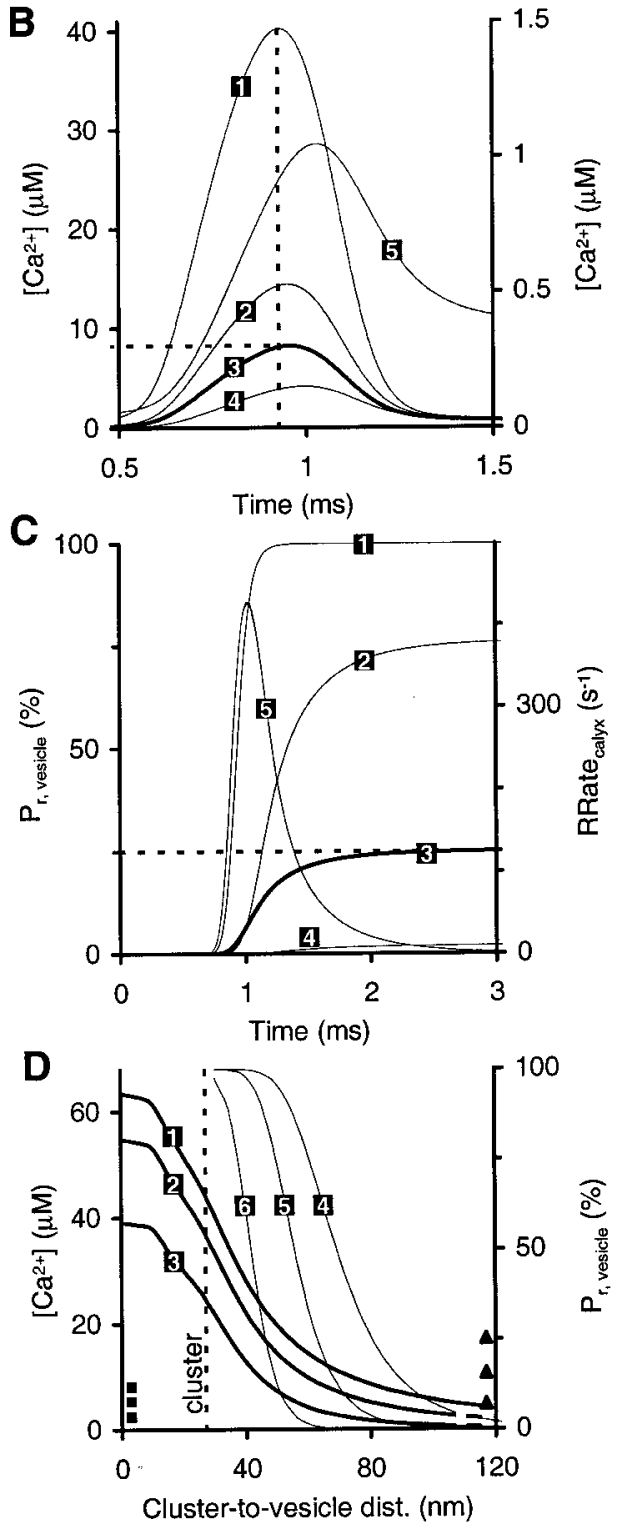

Figure 7. Heterogeneity of AP-evoked $\left[\mathrm{Ca}^{2+}\right]$ transients and their effects on release probability. $A$, Reaction volume with the reference topography used in the simulation; all numbers are given in nanometers (not drawn to scale). Partial 5 and $20 \mathrm{~nm}$ grids indicate varying spatial resolution (see Materials and Methods). Shown is a $\mathrm{Ca}^{2+}$ channel cluster (12 channels; in first voxel layer on membrane) and readily releasable vesicles (three examples shown) on the AZ; AZs have variable size. The illustration shows one example of random placement of a cluster and vesicles at a single AZ. $B$, Time course of $\left[\mathrm{Ca}^{2+}\right]$ for vesicles at different distances from the channel cluster. Trace 1,30 nm from the center of the cluster. Trace 2, $60 \mathrm{~nm}$ from the center of the cluster. Trace 3, Average over all vesicles; peak, $8.2 \mu \mathrm{M}$, half-width, $391 \mu$ sec. Trace 4, $120 \mathrm{~nm}$ icles and their $\mathrm{Ca}^{2+}$ sources; different distributions of cluster-tovesicle distances may result in similar net $P_{\mathrm{r} \text {, calyx }}$ and thus may reproduce the experimental data equally well. Below, we propose a possible specific topography, which is consistent with the above properties and the anatomic data. Using this topography in the model, we simulate the physiological $\left[\mathrm{Ca}^{2+}\right]$ transients that control phasic transmitter release at AZs.

\section{Proposed location of $\mathrm{Ca}^{2+}$ channels}

At each AZ a single cluster of $\mathrm{Ca}^{2+}$ channels controls phasic release. The cluster has a diameter of $\sim 50 \mathrm{~nm}$. It consists of 12 channels, with a conductance of $1.2 \mathrm{pS}$ per channel. The $1.2 \mathrm{pS}$ corresponds to an average $i_{\mathrm{Ca}}$, peak $=0.055 \mathrm{pA}$ per channel during APs $\left(p_{\text {open }}=69 \%\right) \cdot \mathrm{Ca}^{2+}$ channels in the cluster are located as was shown in Figure $6 C(N=12)$. The cluster (center) is located at random anywhere in each $\mathrm{AZ}$ (Fig. $7 A$ ). Consistent with anatomical data, individual AZs are circular areas of variable size, with a radius varying around $r_{\text {average }}=125 \mathrm{~nm}, \sigma=31 \mathrm{~nm}$ (distribution of radius is Gaussian; see Table 1).

\section{Proposed location of readily releasable vesicles}

Readily releasable vesicles are located at random anywhere on every AZ, except for whichever space is already occupied by the channel cluster (Fig. 7A). The random location of vesicles was chosen for the lack of direct evidence of a more defined spatial organization. As a consequence of the large AZs, the distance between a vesicle and the center of the channel cluster ranges between 30 and $300 \mathrm{~nm}($ mode $=90 \mathrm{~nm}$; mean $=118$ $\mathrm{nm} ; \sigma=59 \mathrm{~nm})$.

The above topography was used for all further simulations (henceforth reference topography). All numbers given in the following text apply to simulations that use Release Model A, unless indicated otherwise. For the reference topography, uniform $i_{\mathrm{Ca}}$ mode and stochastic $i_{\mathrm{Ca}}$ mode predict almost the same $\left[\mathrm{Ca}^{2+}\right]$ transients and time course of release. Small differences in predicted $P_{\mathrm{r} \text {, calyx }}$ between the two simulation modes are indicated in the corresponding figures.

\section{Heterogeneous release probability and physiological $\mathrm{Ca}^{2+}$ signaling}

In the simulations for the reference topography, vesicles located at different distances from $\mathrm{Ca}^{2+}$ channels are exposed to predicted $\left[\mathrm{Ca}^{2+}\right]$ transients of different amplitude and time course (Fig. 7B). For single APs under physiological conditions, $\left[\mathrm{Ca}^{2+}\right]_{\text {vesicle }}$ varies between $40 \mu \mathrm{M}$ for vesicles closest to the channels (30 $\mathrm{nm}$ from cluster center) and $0.5 \mu \mathrm{M}$ for the very few vesicles furthest away (300 $\mathrm{nm}$ from cluster center) [between 110

from the center of the cluster. Trace 5, Volume average $\left[\mathrm{Ca}^{2+}\right]($ right axis). Vertical dashed line indicates time of peak $I_{\mathrm{Ca}}$. $C$, Time course of cumulative release probability for single vesicle $\left[P_{\mathrm{r} \text {, vesicle }}(t)\right]$ at different distances (same simulation as in $B$ ). 1, 2, 4, Same distances as in $A$. 3,

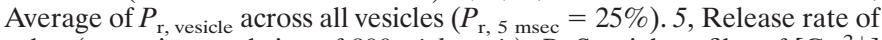
calyx (assuming pool size of 800 ; right axis). $D$, Spatial profiles of $\left[\mathrm{Ca}^{2+}\right]$ (1-3, snapshot at time of peak $I_{\mathrm{Ca}}$ ) and of $P_{\mathrm{r} \text {, vesicle }}(4-6$, after $5 \mathrm{msec}$; right axis) under different buffer conditions [channel cluster centered at distance $0 \mathrm{~nm}$, extending to distance $25 \mathrm{~nm}$ (vertical dashed line)]. 1, 4, Endogenous fixed buffer (EFB) and ATP (control condition). 2, 5, EFB, ATP, and 10 mM EGTA. 3, 6, EFB, ATP, and 1 mm BAPTA. Squares at left axis indicate average $\left[\mathrm{Ca}^{2+}\right]$ across all vesicles $(8.1,5.4$, and $2.5 \mu \mathrm{M}$ for control, EGTA, and BAPTA, respectively). Triangles at right axis indicate average $P_{\mathrm{r} \text {, vesicle }}$ across all vesicles (equaling $P_{\mathrm{r} \text {, calyx }} ; 25,16$, and $7.1 \%$ for control, EGTA, and BAPTA, respectively). 
A

Figure 8. Visualization of reference topography and $\mathrm{Ca}^{2+}$ diffusion at calyx. $A$, Presynaptic membrane with one $\mathrm{AZ}$, one $\mathrm{Ca}^{2+}$ channel cluster, and three readily releasable vesicles. Positioning of cluster and vesicles as well as the number of vesicles on $\mathrm{AZ}$ represents one example of random placement at 600 AZs. Vesicles have a diameter of $50 \mathrm{~nm}$ (same in $A-D)$; drawings are to scale. In the example the vesicles are located at 50,80, and $150 \mathrm{~nm}$ from the center of the channel cluster. $B$, Same as $A$ but viewed from the top and with superimposed false color coding of $\left[\mathrm{Ca}^{2+}\right]$ on the membrane around the channel cluster. Concentrations reflect predicted AP-evoked $\left[\mathrm{Ca}^{2+}\right]$ transients under physiological conditions (same simulation as in Fig. $7 B$; time, $0.60 \mathrm{msec}$ ). For optical clarity AZ has been replaced by a dotted circle. Orange/ red codes show $\left[\mathrm{Ca}^{2+}\right]$ domain around the open channels. $C$, Same as $B$ but viewed from the side, with an additional vertical panel to show $\left[\mathrm{Ca}^{2+}\right]$ in the plane perpendicular to the membrane (time, $1.15 \mathrm{msec}$ ). Dashed circle indicates that the vesicle has fused already. $D$, Variable channel-to-vesicle distances result in variable peaks of $\left[\mathrm{Ca}^{2+}\right]$ transients and thus heterogeneous $P_{\mathrm{r} \text {, vesicle. }}$
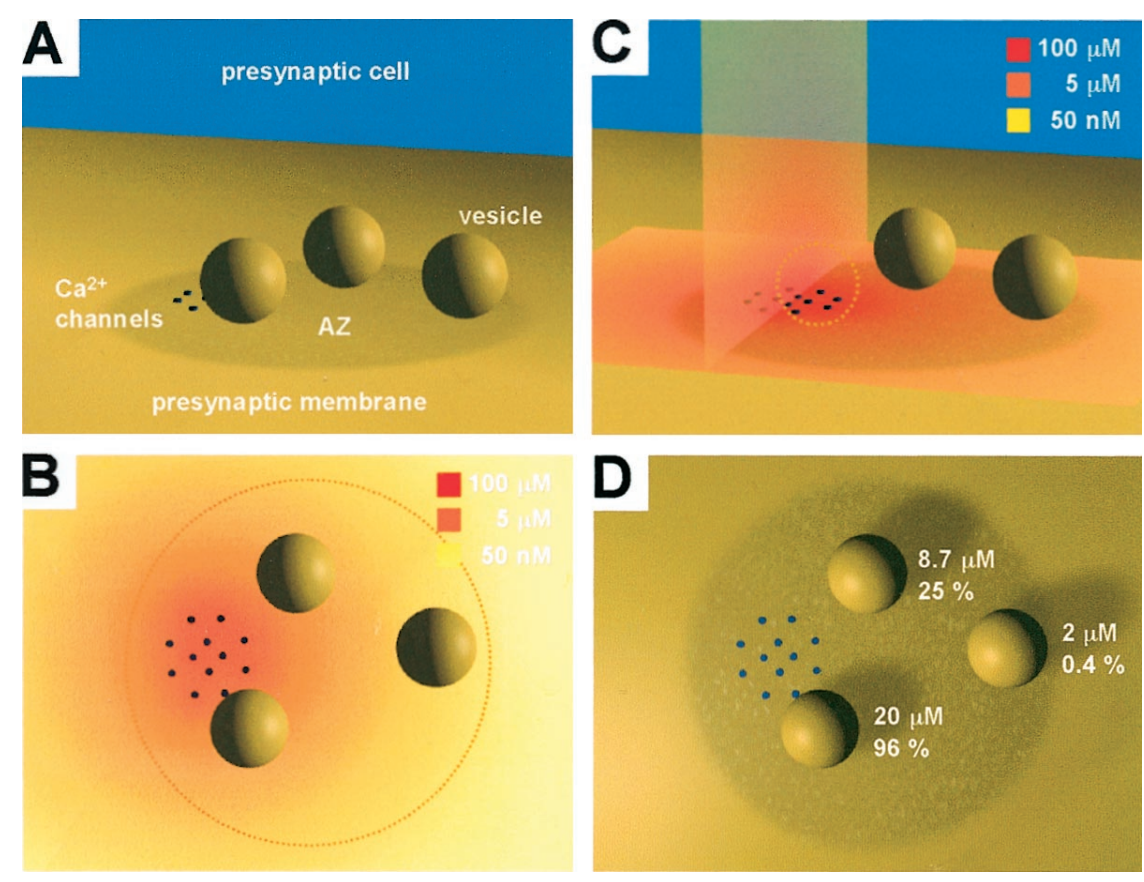

and $1.5 \mu \mathrm{M}$ when using Release Model B]. The peak of the average transient (across all vesicle locations) is $8.2 \mu \mathrm{M}[23 \mu \mathrm{M}]$. It has a half-width (full width at half-maximum) of $391 \mu \mathrm{sec}$ [384 $\mu \mathrm{sec}]\left(\right.$ Fig. $7 B$ ). As a result of the variation in $\left[\mathrm{Ca}^{2+}\right]_{\text {vesicle }}$, $P_{\text {r, vesicle }}$ varies as well, between 100 and $0.006 \%$ [85 and $0.0003 \%$ ] (Fig. $7 C$ ).

In essence, the consideration of variable distance between $\mathrm{Ca}^{2+}$ channels and vesicles constitutes a departure from the concept of a single or average physiological $\left[\mathrm{Ca}^{2+}\right]$ transient driving phasic transmitter release. Likewise, by definition of the nonlinear response of $P_{\mathrm{r} \text {, vesicle }}$ versus $\left[\mathrm{Ca}^{2+}\right]_{\text {vesicle }}$, the definition of an average distance of vesicles to channels is rendered somewhat futile. For the reference topography, the average distance of a vesicle to the center of the channel cluster is $118 \mathrm{~nm}$ (across all vesicles and AZs in the calyx). However, a vesicle actually located at $118 \mathrm{~nm}$ from a cluster does not have the average release probability of all vesicles in the calyx $P_{\mathrm{r} \text {, calyx }}=25 \%$, but only $P_{\mathrm{r} \text {, vesicle }}=2 \%$ (Fig. $7 C$ ). Neither can one define an effective or typical distance. To yield $P_{\mathrm{r} \text {, vesicle }}=P_{\mathrm{r} \text {, calyx }}=25 \%$, a vesicle must be located at $80 \mathrm{~nm}$ from the channel cluster (vesicle at medium distance in Fig. 8). Here, the simulation predicts an approximately bell-shaped transient, peaking at $\left[\mathrm{Ca}^{2+}\right]_{\mathrm{vesicle}}=$ $8.7 \mu \mathrm{M}$ (Fig. 8D, as estimated by Bollmann et al., 2000). However, focusing on the distance of $80 \mathrm{~nm}$ yields misleading results when the effects of added exogenous buffers are interpreted. In the presence of $1 \mathrm{~mm}$ BAPTA the predicted $P_{\mathrm{r} \text {, vesicle }}$ for the vesicle at $80 \mathrm{~nm}$ does not exhibit the reduction of the predicted average release probability to $P_{\mathrm{r} \text {, calyx }}=7 \%$, but to $P_{\text {r, vesicle }}=0.02 \%$.

\section{Heterogeneity and effects of added exogenous buffers}

As shown in Figure $9 A$, the simulation reproduces well (i.e., within \pm 2 SEM confidence interval) the experimentally measured concentration-dependent effects of both BAPTA and EGTA on $P_{\mathrm{r}}$. (The only exception is $P_{\mathrm{r} \text {, calyx }}$ predicted for $1 \mathrm{mM}$ EGTA. Possibly, the proportion of vesicles located away from the channel cluster is larger than that inherent in the random distribution on active zones as used for the reference topography. At
$80 \mathrm{~nm}$ from the cluster, for example, $P_{\mathrm{r} \text {, vesicle }}$ under control conditions is $25 \%$, but $P_{\mathrm{r} \text {, vesicle }}$ under $1 \mathrm{mM}$ EGTA is $20 \%$, i.e., within a \pm 2 SEM confidence interval of the experimental result.)

We will now illustrate why variable location of vesicles predicts $P_{\mathrm{r} \text {, calyx }}$ as observed in the experiments, whereas other, more spatially uniform organizations do not. Variable diff usion distances between release-controlling channel clusters and vesicles create a pool of vesicles with a broad distribution

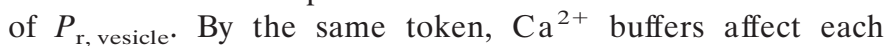
$P_{\mathrm{r} \text {, vesicle }}$ differently, again depending on the diffusion distance for $\mathrm{Ca}^{2+}$ from channels to the particular vesicle. Experiments, however, only measure $P_{\mathrm{r} \text {, calyx }}$, the average of all $P_{\mathrm{r} \text {, vesicle }}$. The overall net effect of added buffers on $P_{\mathrm{r} \text {, calyx }}$, therefore, depends on the distribution of distances between channel clusters and vesicles.

To illustrate the differential efficacy of added buffers, let us consider the vesicles as three separate groups according to distance: close (30-40 nm from cluster center), medium $(40-70 \mathrm{~nm})$, and far $(>70 \mathrm{~nm})$. As was shown in Figure $7 D$, the release probability of close vesicles is $P_{\mathrm{r} \text {, vesicle }}=100 \%$ in response to a single AP (control, i.e., no added exogenous buffers). At 30-40 $\mathrm{nm}$ the exogenous buffers (1 mM BAPTA as well as $10 \mathrm{~mm}$ EGTA) reduce $\left[\mathrm{Ca}^{2+}\right]_{\text {vesicle }}$ to $\sim 0.5$ of that under control conditions. However, $P_{\mathrm{r} \text {, vesicle }}$ of the close vesicles remains unaffected, because release already is saturated. This is different for vesicles at medium distance. Again in a comparison of buffer versus control condition, BAPTA reduces $\left[\mathrm{Ca}^{2+}\right]_{\text {vesicle }}$ to 0.5 of that under control condition, enough to reduce the release probability strongly. However, EGTA at this distance reduces $\left[\mathrm{Ca}^{2+}\right]_{\text {vesicle }}$ less, and $P_{\mathrm{r} \text {, vesicle }}$ stays high. For far vesicles, finally, both buffers strongly reduce $\left[\mathrm{Ca}^{2+}\right]_{\text {vesicle }} . P_{\mathrm{r} \text {, vesicle }}$ is reduced even more strongly because the release-controlling $\mathrm{Ca}^{2+}$ sensor, which at this distance is not saturated, responds steeply to changes in $\left[\mathrm{Ca}^{2+}\right]_{\text {vesicle }}$. Because different vesicles are affected to different degrees, the overall effects of the buffers on $P_{\mathrm{r} \text {, calyx }}$ are in agreement with the experiments, although no single vesicle alone reflects the changes to $P_{\mathrm{r} \text {, calyx }}$. The above reference topography is 


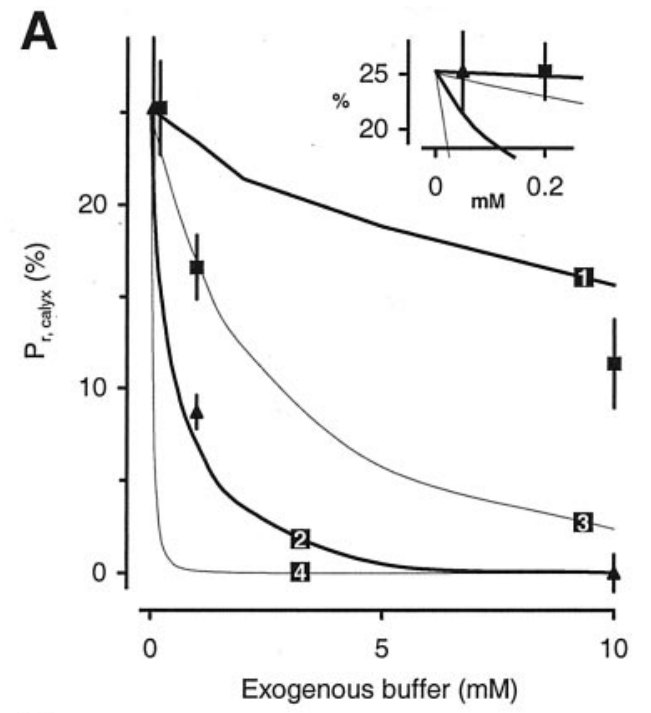

B

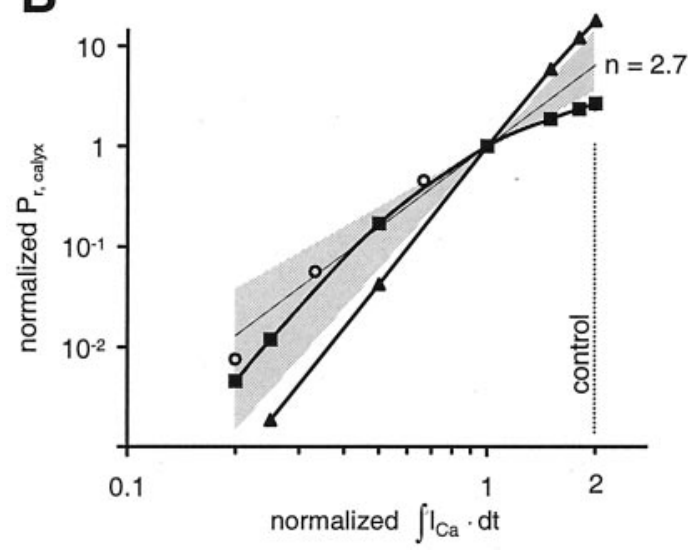

Figure 9. Predicted effects of added exogenous buffers and lowered extracellular $\left[\mathrm{Ca}^{2+}\right]$ on $P_{\mathrm{r} \text {, calyx }}$. $A$, Predicted effect of exogenous buffers on $P_{\mathrm{r}, \text { calyx }}$ for two topographies (reference topography versus periodic grid topography, with $d=60 \mathrm{~nm}$ ) compared with experimental data. Filled squares, EGTA (experimental); filled triangles, BAPTA (experimental; mean \pm SEM as shown in Table 2). Trace 1, EGTA (reference topography); Trace 2, BAPTA (reference topography); Trace 3, EGTA (grid topography); Trace 4, BAPTA (grid topography). Reaction volume and location of channels/vesicles are as in Figure $7 A$ (reference topography) or Figure $4 A$ (grid topography). The same simulations with the stochastic $i_{\mathrm{Ca}}$ mode (data not shown) yielded the same results as the uniform $i_{\mathrm{Ca}}$ mode: EGTA $(1 \mathrm{~mm}$, $24.3 \% \pm 0.5 \% ; 10 \mathrm{~mm}, 16.0 \% \pm 0.3 \%)$ and BAPTA ( $1 \mathrm{~mm}, 7.5 . \% \pm 0.3 \%$; $10 \mathrm{~mm}, 0.1 \% \pm 0.01 \%$; mean \pm SEM after $100-200$ Monte Carlo simulations). $B$, Predicted $P_{\mathrm{r}, \text { calyx }}$ as a function of $\mathrm{Ca}^{2+}$ influx for the same topographies as in $A$ compared with experimental data (see Table 2). Influx was reduced by reducing the single channel conductance from $1.2 \mathrm{pS}$ (control; vertical dashed line) to $0.12 \mathrm{pS}$. Squares (uniform $i_{\mathrm{Ca}}$ mode) and circles (mean of stochastic $i_{\mathrm{Ca}}$ mode) indicate the reference topography (circles are not plotted where indistinguishable from squares; SEMs, after 200 Monte Carlo simulations, are smaller than the dimension of the circles). Triangles (uniform $i_{\mathrm{Ca}}$ mode) indicate the grid topography (predicted slope, $n=4.4$ ). Solid thin line indicates slope, $n=2.7$; shaded area indicates range of slopes in experimental studies $(n=2.2-3.5)$. Each data set is normalized to $(1,1)$. Reaction volume and location of channels/vesicles are as in Figure $7 A$ (reference topography) or Figure $4 A$ (grid topography). $P_{\mathrm{r} \text {, calyx }}$ at control conditions is $25 \%$.

in contrast to the more homogenous periodic grid topography used to infer Property II. While the reduction of the average $\left[\mathrm{Ca}^{2+}\right]_{\text {vesicle }}$ by added exogenous buffers in the grid topography is similar to that in the reference topography, the predicted reduc- tion of $P_{\mathrm{r} \text {, calyx }}$ in the grid topography is much too strong (Figs. $4 B, 7 D)$. In essence, the nonuniformity of vesicle distances inherent in the reference topography creates a safe harbor for some of the vesicles (independent of which release model is used), such that they are released even if the $\mathrm{Ca}^{2+}$ transients are reduced by exogenous buffers.

\section{Heterogeneity and effects of reduced $\mathrm{Ca}^{2+}$ influx}

As described in Property III, the number and location of $\mathrm{Ca}^{2+}$ channels relative to vesicles affect the measured steepness $(m, n)$ at which $P_{\mathrm{r}, \text { calyx }}$ responds to changes in $\mathrm{Ca}^{2+}$ influx. Figure $9 B$ shows the predicted reduction in $P_{\mathrm{r} \text {, calyx }}$ when the $\mathrm{Ca}^{2+}$ influx is reduced by reducing the single channel conductance (1.2 to 0.12 $\mathrm{pS}$, reflecting a reduction from 2 to $\sim 0.2 \mathrm{mM} \mathrm{Ca}^{2+}$ in the extracellular solution). Predicted $P_{\mathrm{r} \text {, calyx }}$ depends supralinearly on the total $\mathrm{Ca}^{2+}$ influx. The predicted slope in the double logarithmic plot increases with decreasing conductance. The average predicted slope is $n=2.8$ (in agreement with the experiments; see Table 2). When $\mathrm{Ca}^{2+}$ influx is reduced by reducing the channel open probability, $P_{\mathrm{r} \text {, calyx }}$ is reduced supralinearly with $m=2.3 \pm 0.1$ (mean \pm SEM after 400 Monte Carlo simulations, as was shown for $N=12$ channels in Fig. $6 B$ ). Note that the prediction for $m$, as well as that for $n$ at large conductance, is somewhat lower than that observed in the experiments. The reason is that $P_{\mathrm{r} \text {, vesicle }}$ of some vesicles is almost saturated and, therefore, less sensitive to changes in $\left[\mathrm{Ca}^{2+}\right]_{\text {vesicle }}$. For comparison, the predicted $n$ for the periodic grid topography $(d=60 \mathrm{~nm})$ is 4.4 , i.e., outside the range observed in the experiments (Fig. 9B).

\section{Functional significance of proposed topography for high-frequency transmission}

Contribution of $\mathrm{Ca}^{2+}$ diffusion to synaptic delay

The time of $\mathrm{Ca}^{2+}$ diff usion from channels to vesicles is believed to constitute only a small part of synaptic delay times (Adler et al., 1991; Yamada and Zucker, 1992; Borst and Sakmann, 1996). Our simulations confirm this view for the calyx, albeit with some qualifications (simulations used Release Model A; analogous simulations that used Release Model B yielded similar results; data not shown).

Once $\mathrm{Ca}^{2+}$ channels have opened, $\mathrm{Ca}^{2+}$ needs to diffuse to the vesicle (i.e., to its presumably colocalized release-controlling $\mathrm{Ca}^{2+}$ sensor). This process is not instantaneous and thus contributes to synaptic delay. To quantify the effect, Figure $10 \mathrm{~A}$ shows the time course of $\mathrm{Ca}^{2+}$ influx through channels, superimposed with the predicted time course of $\left[\mathrm{Ca}^{2+}\right]$ transients at various distances. As shown for the case of a nonphysiological pulse-like whole-cell $\mathrm{Ca}^{2+}$ current $\left(I_{\mathrm{Ca}}\right.$, pulse $)$, the local transient at the channel cluster (30 $\mathrm{nm}$ from center) reaches nearly steady state within $\sim 100 \mu \mathrm{sec}$ after onset of the $\mathrm{Ca}^{2+}$ current. In contrast, the average transient (average across all vesicles) as well as the transient at $200 \mathrm{~nm}$ has not yet reached its steady-state level. In other words, because of buffered diffusion of $\mathrm{Ca}^{2+}$ the time course of $\left[\mathrm{Ca}^{2+}\right]$ transients controlling the vesicles would be delayed against $I_{\mathrm{Ca}}$ if it were pulse-like.

This is different in the case of a physiological, approximately bell-shaped $I_{\mathrm{Ca}}\left(I_{\mathrm{Ca} \text {, physiol }}\right)$ as generated by a cluster of $\mathrm{Ca}^{2+}$ channels that open and close stochastically (Fig. $10 \mathrm{~B}$; time integrals of $I_{\mathrm{Ca} \text {, pulse }}$ and $I_{\mathrm{Ca} \text {, physiol }}$ are the same). The local $\left[\mathrm{Ca}^{2+}\right]$ transient at the channel cluster follows $I_{\mathrm{Ca}}$, physiol almost perfectly (data not shown in Fig. $10 B$ ). Even at $200 \mathrm{~nm}$, the peak of $\left[\mathrm{Ca}^{2+}\right]$ is reached only $\sim 150 \mu \mathrm{sec}$ later than the peak of $I_{\mathrm{Ca}}$, physiol $i t s e l f$. The average $\left[\mathrm{Ca}^{2+}\right]$ transient follows the time course of $I_{\mathrm{Ca}}$, physiol 

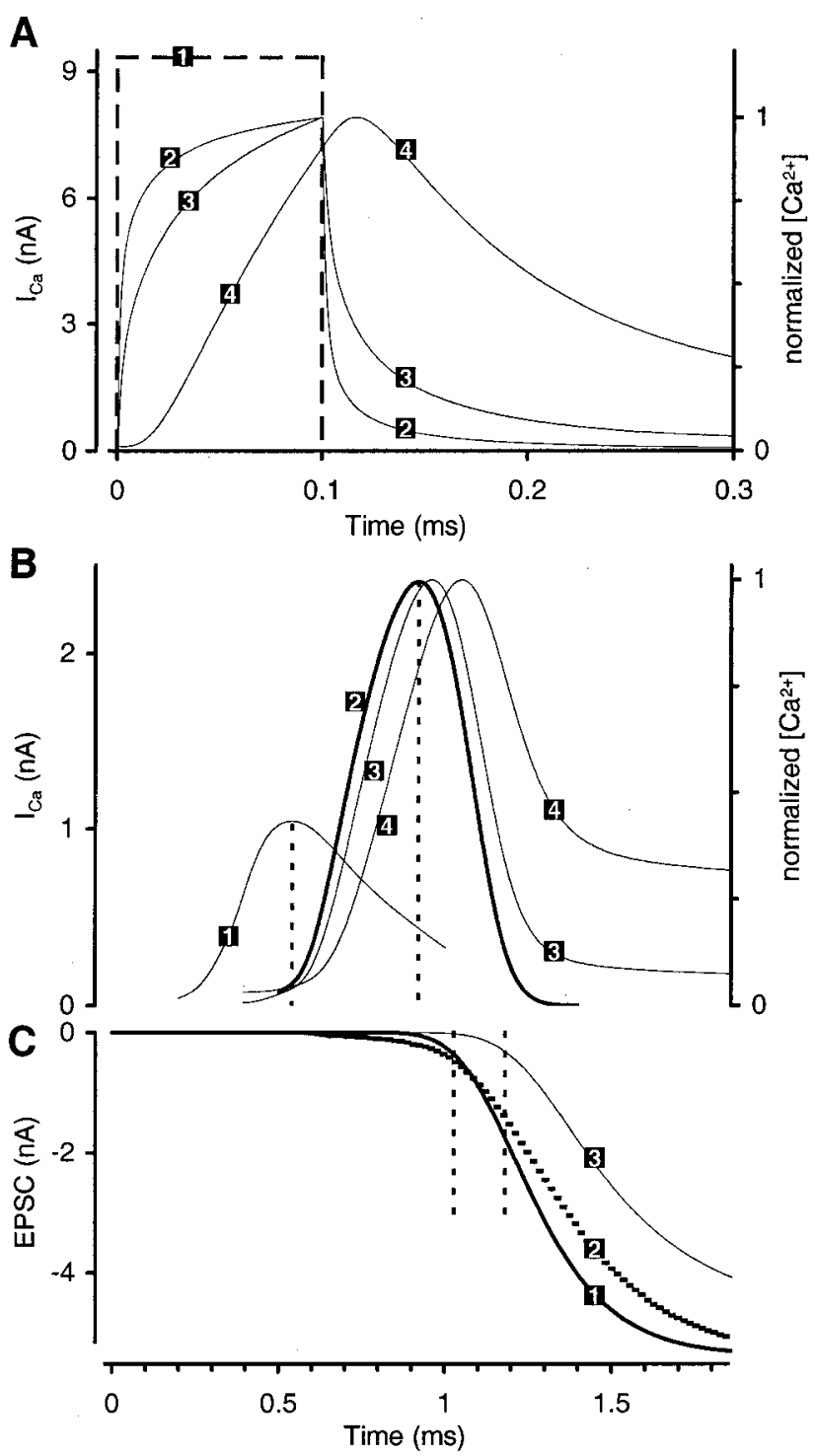

Figure 10. Functional significance of variable channel-to-vesicle distance: synaptic delay. $A$, Pulse-like $I_{\mathrm{Ca}}$ (dashed line) generates $\left[\mathrm{Ca}^{2+}\right]$ transients with different time courses and amplitudes at different distances from channel cluster (solid lines, right axis; each normalized to the same amplitude). Reaction volume and topography are the same as in Figure $7 A$. See Results for further details. Trace 1, Rectangular pulse-like $I_{\mathrm{Ca}}$ of $100 \mu \mathrm{sec}$ duration. Trace 2, Distance $30 \mathrm{~nm}\left[\mathrm{Ca}^{2+}\right]_{\text {peak }}=167 \mu \mathrm{M}$. Trace 3 , Average across vesicles, $\left[\mathrm{Ca}^{2+}\right]_{\text {peak }}=32 \mu \mathrm{M}$. Trace 4, Distance $200 \mathrm{~nm}$ $\left[\mathrm{Ca}^{2+}\right]_{\text {peak }}=3.6 \mu \mathrm{M}$. B, Physiological $I_{\mathrm{Ca}}$ (heavy line) generates $\left[\mathrm{Ca}^{2+}\right]$ transients with different time courses and amplitudes at different distances from channel cluster (thin lines, right axis; each normalized to the same amplitude). Reaction volume and topography are the same as in Figure $7 A$. Trace 1, AP (time course only; same as trace 1 in Fig. $6 A$ ). First dashed line from the left indicates the time of peak of AP $(0.54 \mathrm{msec})$. Trace 2, Physiological whole-cell $I_{\mathrm{Ca}}$. Second dashed line indicates the time of peak of $I_{\mathrm{Ca}}(0.92 \mathrm{msec})$. Trace 3 , Average across vesicles, $\left[\mathrm{Ca}^{2+}\right]_{\text {peak }}=8.2 \mu \mathrm{M}$. Trace 4, Distance $200 \mathrm{~nm}\left[\mathrm{Ca}^{2+}\right]_{\text {peak }}=1.6 \mu \mathrm{M}$. C, For same simulations as in $B$, trace 1 predicted EPSC for physiological $I_{\mathrm{Ca}}$. First vertical dashed line from the left indicates the time of peak of release rate predicted for physiological $I_{\mathrm{Ca}}(1.03 \mathrm{msec})$. Trace 2, Measured single EPSC from Bollmann et al. (2000) (shifted by $\sim 400 \mu \mathrm{sec}$ to the left). Trace 3, EPSC for virtual $\left[\mathrm{Ca}^{2+}\right]$ transient (transient as trace 3 in $B$ ). Second vertical dashed line from the left indicates the time of peak of release rate predicted for virtual transient $(1.18 \mathrm{msec})$. Note that $C$ uses the same time scale as $B$. well, with a small lag of $\sim 50 \mu$ sec (see also Schneggenburger and Neher, 2000). Thus we estimate that during APs at the calyx the direct contribution of $\mathrm{Ca}^{2+}$ diff usion time to synaptic delay is only $\sim 50 \mu \mathrm{sec}$. This is aided by the time course of the physiological $\mathrm{Ca}^{2+}$ current. Its rise and decay [approximately bell-shaped, half-width (full width at half-maximum), $383 \mu \mathrm{sec}$ ] are slow enough to be followed easily by the buffered diffusion system that governs the $\left[\mathrm{Ca}^{2+}\right]$ transients.

We will now consider another effect of $\mathrm{Ca}^{2+}$ diffusion on synaptic delay. We define synaptic delay as the time between the onset ( $5 \%$ of peak) of $I_{\mathrm{Ca}}$ and the onset of the predicted EPSC. The delay time in the model does not represent synaptic delay as it is measured in experiments. This is because the model does not account for additional delays caused by steps after the response of the release-controlling $\mathrm{Ca}^{2+}$ sensor. Therefore, the experimentally measured EPSC in Figure $10 C$ (dashed curve) was shifted in time ( $\sim 400 \mu \mathrm{sec}$ toward earlier times) to allow for comparison of the time course (Bollmann et al., 2000). [Note, however, that the EPSC time course predicted by the model cannot be expected to match perfectly that of measured EPSCs. First, the postsynaptic response to transmitter deviates from a linear superposition of quantal EPSCs (Sakaba and Neher, 2001b), a fact not yet included in the model. Second, additional steps in the transmission process after fusion of a vesicle may contribute to the variation in the time of onset of quantal EPSCs.]

In the following analysis we will focus on differences in synaptic delay predicted for different $\left[\mathrm{Ca}^{2+}\right]$ transients rather than on delay times in absolute terms. The onset of the EPSC for the pulse-like $I_{\mathrm{Ca}}$ is at $65 \mu \mathrm{sec}$ after the onset of $I_{\mathrm{Ca}}(20-80 \%$ rise time of EPSC is $230 \mu \mathrm{sec}$; peak is $7.1 \mathrm{nA}$; data not shown). The short delay is expected from the high $\left[\mathrm{Ca}^{2+}\right]$ transients and the fast forward binding rates of the $\mathrm{Ca}^{2+}$ sensor, although the $\mathrm{Ca}^{2+}$ does not reach all vesicles immediately. For the physiological $I_{\mathrm{Ca}}$, the onset of the EPSC (Fig. 10C, trace 1; rise time, $310 \mu \mathrm{sec}$; peak, $5.3 \mathrm{nA}$ ) is $\sim 400 \mu \mathrm{sec}$ after the onset of $I_{\mathrm{Ca}}$ (onset of $I_{\mathrm{Ca}}$, physiol is at the peak of the AP, at $540 \mu \mathrm{sec})$. The peak of the average release rate underlying the EPSC (data not shown) is at $1030 \mu \mathrm{sec}$ or $100 \mu \mathrm{sec}$ later than the peak of $I_{\mathrm{Ca}}$, physiol (Fig. 10C, first vertical dashed line from the left). For the calyx this time interval has been measured to be approximately five times larger ( $\sim 500 \mu \mathrm{sec}$; Borst and Sakmann, 1996). This confirms that a large part of the physiological delay is caused by steps after the kinetic response of the release-controlling $\mathrm{Ca}^{2+}$ sensor.

Bollmann et al. (2000) and Schneggenburger and Neher (2000) have shown that EPSCs at the calyx, when evoked by $\left[\mathrm{Ca}^{2+}\right]$ uncaged from photosensitive chelators, have synaptic delays which are shorter, the higher the $\left[\mathrm{Ca}^{2+}\right]_{\text {vesicle. In the model, }}$ phasic release comprises release from vesicles that are located at different distances to channel clusters and thus are exposed to $\left[\mathrm{Ca}^{2+}\right]$ transients of different amplitude (maximum of $\sim 40$ vs $\sim 8$ $\mu \mathrm{M}$ for the average). Therefore, the vesicles in close proximity to channel clusters release neurotransmitter earlier than the average vesicle and thus shorten the synaptic delay. To quantify the effect, we calculated the EPSC that would be predicted if all vesicles were controlled by a single virtual $\left[\mathrm{Ca}^{2+}\right]$ transient; i.e., if all vesicles were located at the same distance from $\mathrm{Ca}^{2+}$ channel clusters. As the virtual transient, we chose the average $\left[\mathrm{Ca}^{2+}\right](t)$ predicted by the diffusion model (average across all vesicles) (Fig. $10 B)$. This simulation essentially removes effects of heterogeneous $\left[\mathrm{Ca}^{2+}\right]_{\text {vesicle }}$ from the predicted EPSC. The resulting EPSC $\left(\right.$ EPSC $_{\text {virtual }}$ ) (Fig. $10 C$, trace 3 ) has a peak amplitude of $4.5 \mathrm{nA}$ (rise time, $410 \mu \mathrm{sec}$ ), similar to that of $\mathrm{EPSC}_{\text {physiol }}$ predicted by 
the full diffusion model. However, the onset of EPSC $_{\text {virtual }}$ is $\sim 150 \mu \mathrm{sec}$ later than that of $\mathrm{EPSC}_{\mathrm{physiol}}$. The peak of the vesicle release rate generating $\mathrm{EPSC}_{\text {virtual }}$ is at $1182 \mu \mathrm{sec}$ (Fig. $10 \mathrm{C}$, second vertical dashed line from the left).

In summary, the diffusion time of $\mathrm{Ca}^{2+}$ contributes only $\sim 50$ $\mu$ sec to the synpatic delay. However, a direct contribution of only $\sim 50 \mu$ sec does not imply that buffered $\mathrm{Ca}^{2+}$ diffusion is insignificant for determining the onset of EPSCs. Instead, the time of onset of physiological EPSCs depends on the amplitude of $\left[\mathrm{Ca}^{2+}\right]$ transients and thus on where the vesicles are located with respect to $\mathrm{Ca}^{2+}$ channels.

\section{Invariance of release time course}

The time course of phasic transmitter release has been shown to be remarkably stable, even when the rate of release is reduced by several orders of magnitude, for example by lowering $\left[\mathrm{Ca}^{2+}\right]$ of the extracellular solution from $2 \mathrm{~mm}$ (control) to $0.25 \mathrm{~mm}$ (Van der Kloot, 1988; Isaacson and Walmsley, 1995; Borst and Sakmann, 1996). To test the compliance of the model with this finding, we reduced the single channel conductance in the above simulation to $0.15 \mathrm{pS}$ per channel, a value eight times lower than that used for the above EPSC $_{\text {physiol }}$. The predicted $P_{\mathrm{r} \text {, calyx }}$ is $0.11 \%$, which corresponds to less than one vesicle per AP (uniform $i_{\mathrm{Ca}}$ mode). The simulated EPSC $\left(\mathrm{EPSC}_{\mathrm{low}} \mathrm{Ca}\right.$; data not shown) thus represents the average of evoked, quantal miniature EPSCs.

The predicted relative time course of $\mathrm{EPSC}_{\text {low Ca }}$ is similar to that of EPSC $_{\text {physiol }}$ [Release Model A, rise time $415 \mu \mathrm{sec}$ (low $\mathrm{Ca}^{2+}$ ) vs $310 \mu \mathrm{sec}$ (physiol); Release Model B, rise time $310 \mu \mathrm{sec}$ (low $\mathrm{Ca}^{2+}$ ) vs $295 \mu \mathrm{sec}$ (physiol)]. The onset of EPSC $_{\text {low Ca }}$ is later than that of $\mathrm{EPSC}_{\text {physiol }}$ (Release Model A, +135 $\mu \mathrm{sec}$; Release Model B, $+80 \mu \mathrm{sec})$, a prediction consistent with a measured increase in delays after a reduction of $\mathrm{Ca}^{2+}$ influx at the calyx (Taschenberger and von Gersdorff, 2000).

The small increase in the predicted delay of EPSC $_{\text {low Ca }}$ versus EPSC $_{\text {physiol }}$ does not (primarily) stem from the lower $\left[\mathrm{Ca}^{2+}\right]$ transients reaching the vesicles any later. The (nonuniform) $\left[\mathrm{Ca}^{2+}\right]$ transients for the low $\mathrm{Ca}^{2+}$ condition have amplitudes approximately eight times smaller, but their time courses differ by at most $\pm 20 \mu \mathrm{sec}$ from those under control conditions (data not shown). Rather, the increase stems from the kinetic response of the molecular release model itself. Both models (A and $\mathrm{B}$ ) predict slower responses to the $\left[\mathrm{Ca}^{2+}\right]$ transients of the low $\mathrm{Ca}^{2+}$ condition. Still, the overall changes in EPSC time course are small. This is because, under the low $\mathrm{Ca}^{2+}$ condition, the EPSC is generated almost exclusively by release from vesicles located close to the channel clusters (30 $\mathrm{nm}$ from cluster center). Here, predicted $\left[\mathrm{Ca}^{2+}\right]$ transients are still as high as $5 \mu \mathrm{M}$ (Release Model $\mathrm{B}, 14 \mu \mathrm{M})$, a concentration in which the kinetic response of both release models is similar to that under control conditions (Bollmann et al., 2000; Schneggenburger and Neher, 2000).

In summary, the predicted invariance of the (relative) release time course for reduced $\mathrm{Ca}^{2+}$ influx depends not only on the release model but on the release site topography as well.

\section{Synaptic reliability during consecutive APs}

Part of a structure involved in the location of sound, the calyx is capable of sustained high-frequency transmission with high temporal fidelity between presynaptic and postsynaptic AP (Oertel, 1999). Possible functional advantages of heterogeneous release probability with respect to this function have been discussed by Wu and Borst (1999) and Sakaba and Neher (2001b). Briefly, one concept proposes that vesicles that, by whatever mechanism, have a low probability to be released by a single AP may have a higher release probability in subsequent, consecutive APs $(\sim 100 \mathrm{~Hz}$ or more). The increase in release probability is thought to be attributable to the $\mathrm{Ca}^{2+}$ (bound or unbound) that remains in the calyx from the influx generated by one or more previous APs. The exact mechanism of this facilitation is not crucial to the concept (for an overview, see Fisher et al., 1997).

In the model calyx the release probability is heterogeneous because of a nonuniform location of vesicles across different release sites of the same calyx. We will show how this leads to a gradual increase in release probability during consecutive APs, simulated as a $100 \mathrm{~Hz}$ train of 10 APs. Two situations are compared: (1) release predicted for the native calyx, i.e., with only endogenous buffers in the presynaptic solution (henceforth "native calyx") and (2) release predicted for a calyx dialyzed with 0.5 mm EGTA ("EGTA calyx"). To include the effect of $\mathrm{Ca}^{2+}$ removal, we added to the model a linear extrusion mechanism with a rate $\gamma=400 / \mathrm{sec}$ (Helmchen et al., 1997) (see Materials and Methods). $I_{\mathrm{Ca}}$ during individual APs remained unchanged. This is in line with the above concept, which assumes that changes in $I_{\mathrm{Ca}}$ during consecutive APs are not the main factor for increasing $P_{\mathrm{r}}$.

Because the model simulates the $\mathrm{Ca}^{2+}$ dynamics of the entire calyx, it predicts a rise in the volume average $\left[\mathrm{Ca}^{2+}\right]$ during each AP (Fig. 11 $A$ ). Figure $11 B$ shows the predicted phasic transmitter release. In the following, $P_{\mathrm{r}}(i)_{\text {cumu }}$ denotes the cumulative number of vesicles released up to the $i$ th $\mathrm{AP}[\mathrm{AP}(i)]$ divided by the total number of readily releasable vesicles at the start of the train. Comparing the incremental release from AP to AP, we extract the release probability per single AP $\left[P_{\mathrm{r}}(i)_{\text {single }}\right]$, which denotes the number of vesicles released by $\mathrm{AP}(i)$ divided by the total number of vesicles available for release before $\operatorname{AP}(i) . P_{\mathrm{r}}(i)_{\text {single }}$ is thus the average release probability of those vesicles that have remained in the calyx after all APs before $\mathrm{AP}(i)$. Note that the EPSCs in Figure $11 C$ are calculated by assuming a linear superposition of quantal EPSCs (see Materials and Methods). This leads to a deviation from experimental EPSCs, particularly during the decay phase of single EPSCs and during trains (Sakaba and Neher, 2001b). Therefore, we focus our analysis on predicted release probabilities but nevertheless include the EPSCs for illustration of the concept.

For the EGTA calyx the fraction of released vesicles increases to $P_{\mathrm{r}}(10)_{\text {cumu }}=49.3 \%$, meaning that after the 10th AP approximately one-half of the readily releasable vesicles in the original calyx have been released. The predicted $P_{\mathrm{r}}(i)_{\text {single }}$ decreases for consecutive APs because the release probability is heterogeneous. During each AP the vesicles located closer to the channel cluster are released preferentially, whereas the vesicles further away tend to be left behind. Such a decrease in $P_{\mathrm{r}}(i)_{\text {single }}$ during the first few stimuli of a train has been observed at the calyx (Sakaba and Neher, 2001b). The predicted volume-averaged $\left[\mathrm{Ca}^{2+}\right]$ rises only moderately during the course of 10 APs: from $0.18 \mu \mathrm{M}$ after the first AP (near spatial equilibrium) to $0.40 \mu \mathrm{M}$ after the tenth AP. Therefore, in line with the above concept, facilitation is kept to a minimum and the increase in $P_{\mathrm{r}}(i)_{\text {single }}$ during the train of APs does not occur in the EGTA calyx.

For the native calyx the situation is quite different. $P_{\mathrm{r}}(i)_{\mathrm{cumu}}$ increases to $P_{\mathrm{r}}(10)_{\text {cumu }}=98.9 \%$. In contrast to the EGTA calyx, the volume-averaged $\left[\mathrm{Ca}^{2+}\right]$ rises significantly from $0.34 \mu \mathrm{M}$ after the first AP to $2.4 \mu \mathrm{M}$ after the tenth AP. $P_{\mathrm{r}}(i)_{\text {single }}$ decreases from the first to the third AP. However, after the third AP the release probability increases again, to $P_{\mathrm{r}}(10)_{\text {single }}=62.1 \%$. The gradual 

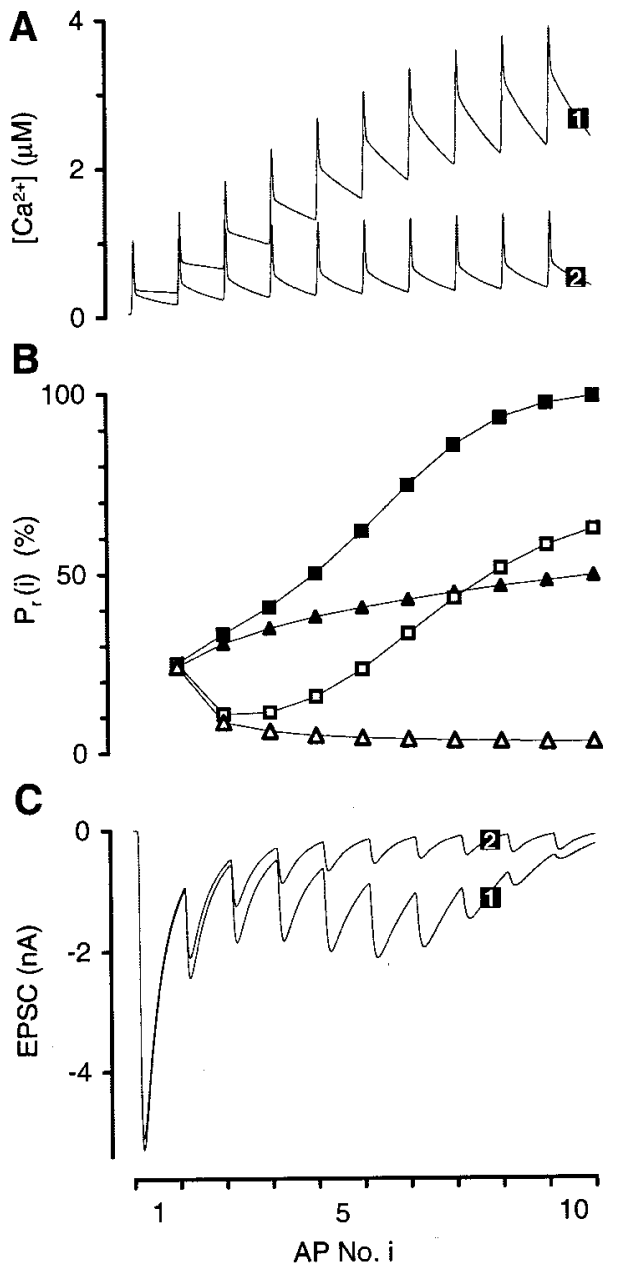

Figure 11. Functional significance of variable channel-to-vesicle distance: consecutive APs. $A$, Volume average $\left[\mathrm{Ca}^{2+}\right]$ in calyx during 10 consecutive APs at $100 \mathrm{~Hz}$ (reaction volume as in Fig. $7 A$, reference topography; $\mathrm{Ca}^{2+}$ extrusion included; for details, see Results). Trace 1 shows native calyx (endogenous fixed buffer and ATP only). Trace 2 shows EGTA calyx (with added $0.5 \mathrm{~mm}$ EDTA). $B$, Same simulation as in $A$. Filled squares, Cumulative number of vesicles released after each pulse in the native calyx as a fraction of the number of vesicles in the readily releasable pool at the start of the train $\left[P_{\mathrm{r}}(i)_{\mathrm{cumu}}\right]$. Filled triangles, Same as squares but for EGTA calyx. Open squares, Same as filled squares but showing release probability per single AP $\left[P_{\mathrm{r}}(i)_{\text {single }}\right]$. Open triangles, Same as open squares but for EGTA calyx. $C$, Same simulation as in $A$. Trace 1, EPSC for native calyx; trace 2, EPSC for EGTA calyx. Note that $A-C$ use the same time scale $(1 \mathrm{AP}=10 \mathrm{msec})$.

increase in $P_{\mathrm{r}}(i)_{\text {single }}$ allows the model synapse to gradually make use of the vesicles not released during the first APs (because of disadvantageous location). The EPSC amplitude decreases for APs 8, 9, and 10. This is because the original pool of vesicles is almost exhausted. $P_{\mathrm{r}}(10)_{\mathrm{cumu}}=98.9 \%$ implies that the calyx could not maintain transmission beyond $100 \mathrm{msec}$ (10 APs). However, within the first $100 \mathrm{msec}$, experiments for the calyx have measured significant recruitment of new vesicles, i.e., vesicles that were not yet included in the original count of readily releasable vesicles ( $\mathrm{Wu}$ and Borst, 1999). Even if these vesicles had a rather low response to single isolated APs (because of either intrinsic properties or their disadvantageous location), they could be facilitated in the above manner (see discussion in $\mathrm{Wu}$ and Borst, 1999). Therefore, in a real calyx such rapidly recruited new vesicles will be available to maintain the release of neuro- transmitter during consecutive APs (Wang and Kaczmarek, 1998).

\section{Facilitation}

In the native calyx, $P_{\mathrm{r}}(2)_{\text {single }}=10.8 \%$, whereas this probability is lower $(8.5 \%)$ in the EGTA calyx. A lower $P_{\mathrm{r}}(2)_{\text {single }}$ in the presence of buffers, i.e., a more pronounced heterogeneity, has in fact been observed for the calyx (Sakaba and Neher, 2001b). The 10.8 versus $8.5 \%$ implies a facilitation of $P_{\mathrm{r}}$ of $\sim 27 \%$ (comparing the native vs the EGTA calyx). In the model the facilitation of vesicles during consecutive APs is caused by several effects: (1) $\mathrm{Ca}^{2+}$ still bound to binding sites of release-controlling $\mathrm{Ca}^{2+}$ sensor after the first AP (marginal contribution, off-rates are fast); (2) pre-equilibration of the release-controlling $\mathrm{Ca}^{2+}$ sensor to a higher volume average $\left[\mathrm{Ca}^{2+}\right]_{\mathrm{vol}}$ (marginal contribution; on-rates are fast); (3) partial depletion (local and global) of endogenous buffers (fixed and ATP) and therefore less effective attenuation of the local $\left[\mathrm{Ca}^{2+}\right]_{\text {vesicle }}$ for the second AP [moderate contribution because attenuation of $\left[\mathrm{Ca}^{2+}\right]_{\mathrm{vesicle}}$ by endogenous buffers is weak (see Materials and Methods); but see Bennett et al. (2000) and discussion in Neher (1998b)]; (4) higher $\left[\mathrm{Ca}^{2+}\right]_{\text {vesicle }}$ during the second AP because transients induced by $I_{\mathrm{Ca}}$ are added (approximately) to the higher volume-averaged $\left[\mathrm{Ca}^{2+}\right]$ at the beginning of the second AP (main contribution). Note that the above effects may not describe facilitation of physiological release probabilities in a more than qualitative way, and our analysis does not preclude the possibility of additional mechanisms mediating short-term facilitation at the calyx. In experiments with trains of APs, the second EPSC and thus the implied $P_{\mathrm{r}}(2)_{\text {single }}$ is higher than that predicted by our model (BarnesDavies and Forsythe, 1995; Borst et al., 1995; Schneggenburger et al., 1999).

\section{DISCUSSION}

\section{Release site topography}

The main result is the topography of release sites at the calyx (postnatal days 8-10). The majority of $\mathrm{Ca}^{2+}$ channels controlling phasic release is organized in clusters ( 10 or more channels per cluster; cluster diameter $\sim 50 \mathrm{~nm}$ or less). Readily releasable vesicles are located at a variable distance from the cluster controlling their release $(30-300 \mathrm{~nm}$; average $\sim 100 \mathrm{~nm})$. Using this nonuniform topography, the model reproduces $P_{\mathrm{r}}$ in agreement with experiments. This does not exclude possible other distributions of cluster-to-vesicle distances (provided they are not equidistant) because these may yield similar net $P_{\mathrm{r}}$ across the multiple release sites of the calyx. For example, we cannot rule out the possible existence of colocalized subclasses of vesicles for which the distance from channels might be controlled by anatomical links (Rozov et al., 2001; Sakaba and Neher, 2001a).

\section{Robustness of findings on topography}

The findings on the topography are robust; they are based on large quantitative effects and are insensitive to imperfectly known parameters. For example, reproduction of the effects of the buffers on $P_{\mathrm{r}}$ does not depend on the exact distribution of channelto-vesicle distances (see Property I), nor does it depend on the assumed time course of single channel currents [a pulse-like current of $100 \mu \mathrm{sec}$ duration (Fig. 10A) predicts $33 \% P_{\mathrm{r}}$ for the control condition, $21 \%$ for $10 \mathrm{~mm}$ EGTA, and $11 \%$ for $1 \mathrm{~mm}$ BAPTA]. The requirement that neighboring $\mathrm{Ca}^{2+}$ sources be separated by at least $\sim 200 \mathrm{~nm}$ is valid for two parameter sets for EGTA and is independent of ATP (see Property II). The require- 
ment that multiple $\mathrm{Ca}^{2+}$ channels control individual vesicles can be derived independently of $\mathrm{Ca}^{2+}$ diffusion (see Property III). Furthermore, predicted $P_{\mathrm{r}}$, the basis for the findings on topography, shows only weak sensitivity to any reasonable variation of endogenous buffer parameters (see Materials and Methods). Finally, two release models yield the same findings on the topography (see Properties I-III).

\section{Alternative, but less likely, topographies}

Having focused our analysis on required topographic properties rather than on specific fixed topographies, we can evaluate a large parameter space of alternative topographies without testing them in time-consuming simulations. Although conceivable anatomically, alternative topographies satisfy only one or two of the required properties and, therefore, are consistent with only parts of the experimental data.

For example, a vesicle located inside a ring of $\mathrm{Ca}^{2+}$ channels (Bertram et al., 1999) is consistent with the requirement that a vesicle be controlled by multiple $\mathrm{Ca}^{2+}$ channels at similar distance. However, because the channel-to-vesicle distance does not vary across release sites, such a topography is inconsistent with the measured buffer effects.

As another example, it appears unlikely that the majority of AZs is controlled by more than a few $\mathrm{Ca}^{2+}$ channel clusters. More than one cluster per AZ would decrease the nonuniformity of cluster-to-vesicle distances (CV of distances across release sites too low) while also decreasing the uniformity of distances of an individual vesicle to its release-controlling $\mathrm{Ca}^{2+}$ channels.

\section{$\mathrm{Ca}^{2+}$ transients at room temperature}

Because the two release models ascribe different intrinsic $\mathrm{Ca}^{2+}$ sensitivities to a releasable vesicle, predicting exact amplitudes of AP-evoked $\left[\mathrm{Ca}^{2+}\right]$ transients depends on which model is used in the simulation (see Results). As for the time course, the simulations suggest that it follows the time course of $I_{\mathrm{Ca}}$ (half-width $\sim 380 \mu \mathrm{sec}$ ) with only a small lag of $\sim 50 \mu \mathrm{sec}$ (except for the few vesicles located further than $\sim 150 \mathrm{~nm}$ away). Note that, at physiological temperatures, $\left[\mathrm{Ca}^{2+}\right]$ transients reaching vesicles presumably will be briefer and higher, as judged from the changes in $\mathrm{Ca}^{2+}$ influx (Borst and Sakmann, 1998) and APs/EPSCs (Taschenberger and von Gersdorff, 2000).

\section{Release-relevant $\mathbf{C a}^{2+}$ channels and channel subtypes}

For the reference topography, $83 \%$ [Release Model B, 58\%] of whole-cell $I_{\mathrm{Ca}}$ is mediated by $\mathrm{Ca}^{2+}$ channels that are located such that they do not control phasic release directly (see Materials and Methods). This is supported by experiments that show that most $\mathrm{P} / \mathrm{Q}$-type $\mathrm{Ca}^{2+}$ channels appear to be located near release sites, whereas part of the $\mathrm{N}$-type and R-type channels appear to be located several hundreds of nanometers away from AZs (Wu et al., 1999). During development of the calyx (postnatal days 7-13) the contribution of N-type and R-type channels decreases, leaving the $\mathrm{P} / \mathrm{Q}$-type as the predominant $\mathrm{Ca}^{2+}$ channel subtype triggering phasic release (Iwasaki and Takahashi, 1998). We therefore assume that the channel clusters in the model, which mediate the local $\left[\mathrm{Ca}^{2+}\right]$ signal for phasic release, represent $\mathrm{P} / \mathrm{Q}$-type channels.

While channels confined to AZs contribute 17\% [42\%] of the total $\mathrm{Ca}^{2+}$ influx, AZs cover only $2 \%$ of the total presynaptic membrane surface (see Table 1). Assuming similar conductance and $p_{\text {open }}$ for all $\mathrm{Ca}^{2+}$ channels, this implies that the density of channels in AZs is $\sim 10$ times higher than that in the unspecialized membrane [Release Model B, 36 times]. This complies with experiments showing regions of locally elevated $\left[\mathrm{Ca}^{2+}\right]$ at presynaptic release sites (Llinãs et al., 1992; DiGregorio et al., 1999).

\section{Overlap of $\mathrm{Ca}^{2+}$ channel domains and buffer saturation}

Whether phasic transmitter release is controlled by one or by several $\mathrm{Ca}^{2+}$ channel domains per vesicle has been much debated (Augustine, 2001).

Regarding experiments that reduced the open probability of $\mathrm{Ca}^{2+}$ channels, the reference topography reproduces the effects of overlapping domains, as discussed in Borst and Sakmann (1999a). Regarding the effects of added exogenous buffers, vesicles essentially behave as if controlled by a single $\mathrm{Ca}^{2+}$ channel domain. Although the $\mathrm{Ca}^{2+}$ current used in the simulations $\left(I_{\mathrm{Ca} \text {, peak }}=0.66 \mathrm{pA}\right.$ per cluster $)$ is likely too large to be supplied by a single channel, the combined domain of $\sim 10$ clustered channels resembles that of a single large channel. Still, as expected from the relatively low $\left[\mathrm{Ca}^{2+}\right]$ transients (micromolar range), mobile buffers in the simulation deplete only marginally (see Materials and Methods; also see discussion in Naraghi and Neher, 1997).

\section{Possible other causes for heterogeneous $\boldsymbol{P}_{\mathrm{r}}$}

Heterogeneous release probability of vesicles has been observed at several CNS synapses (Dobrunz and Stevens, 1997; Markram et al., 1997; Silver et al., 1998), including the calyx (Sakaba and Neher, 2001b). Even in a readily releasable/fully recovered vesicle pool, a multitude of mechanisms can cause individual vesicles of the pool to have different probabilities of being released during an AP (discussed in Sakaba and Neher, 2001b). We suggest that the observed heterogeneity to a large extent may be caused by the variability in the distance between a vesicle and its releasecontrolling $\mathrm{Ca}^{2+}$ channels; other intrinsic properties of the release apparatus, in particular its sensitivity to local $\left[\mathrm{Ca}^{2+}\right]$ transients, contribute only partially to the heterogeneity. Two arguments support this. (1) $P_{\mathrm{r}}$ in response to spatially uniform $\left[\mathrm{Ca}^{2+}\right]$ signals measured over a wide range of $\left[\mathrm{Ca}^{2+}\right]$ could be well explained by a single kinetic model for all vesicles in the readily releasable pool (Bollmann et al., 2000; Schneggenburger and Neher, 2000). This suggests that, at the calyx, possible intrinsic heterogeneities of the response of the vesicles to $\left[\mathrm{Ca}^{2+}\right]$ are not strongly pronounced (in comparison, see Blank et al., 1998). (2) Although usually not measurable during single APs, the heterogeneity of $P_{\mathrm{r}}$ becomes apparent when the calyx is dialyzed with BAPTA versus EGTA. These $\mathrm{Ca}^{2+}$ buffers differentially modify the $\left[\mathrm{Ca}^{2+}\right]$ transients during APs, the main difference being at what distance from the $\mathrm{Ca}^{2+}$ channels they are most potent in attenuating the $\left[\mathrm{Ca}^{2+}\right]$ transient. Thus it seems likely that the observed heterogeneity of $P_{\mathrm{r}}$ to a large extent is caused by the variability in the distance between vesicles and $\mathrm{Ca}^{2+}$ channels. This assumption accurately predicts the experimental results. Note, however, that these arguments do not preclude possible, additional, intrinsic heterogeneity of $P_{\mathrm{r}}$.

Because other mammalian CNS synapses have similar differential efficacies of BAPTA versus EGTA in suppressing release (Ohana and Sakmann, 1998; Rozov et al., 2001), nonuniform distance between vesicle and $\mathrm{Ca}^{2+}$ source may be found at these synapses as well.

\section{Functional significance of topography and synaptic development}

The rather loose, nonuniform spatial organization of vesicles and $\mathrm{Ca}^{2+}$ channels we propose for the calyx (development stage 
postnatal days $8-10$ ) is in contrast to the spatially more organized release sites of the neuromuscular junction of crayfish (Msghina et al., 1999) and frog (Harlow et al., 2001). However "nonorganized" they may seem, the topographic characteristics we have inferred appear to offer several functional advantages, some of which coincide with synaptic development.

\section{Channel clusters}

The clustering of $\mathrm{Ca}^{2+}$ channels ensures that stochastically gated $\mathrm{Ca}^{2+}$ channels generate a nevertheless reliable and low-noise $\left[\mathrm{Ca}^{2+}\right]$ transient controlling the release of transmitter (discussed in Stanley, 1997). Recent studies at the calyx have found that a further increase in its maximum transmission rate during development (postnatal days 5-14) coincides with a faster time course of the presynaptic AP (Taschenberger and von Gersdorff, 2000). A faster AP, however, only results in a faster $\left[\mathrm{Ca}^{2+}\right]$ transient for all vesicles if the $\mathrm{Ca}^{2+}$ channels are clustered. The clustering of $\mathrm{Ca}^{2+}$ channels (probably P/Q-type; see above) thus ensures maximum benefit of faster APs for synaptic transmission.

\section{Synaptic delay}

Synaptic delay times at the calyx shorten during early development (Taschenberger and von Gersdorff, 2000). The delays depend, among other factors such as AP time course, on the location of vesicles (see Results). Therefore, the shortening of delays may be attributable partly to a possible spatial reorganization of vesicles around release-controlling channels during development.

\section{Heterogeneous $\mathrm{P}_{r}$}

The multitude of channel-to-vesicle distances (in our model, random) serves to create an "immediate back-up pool" of vesicles. Vesicles in this pool, which is defined only by the location of the vesicles, are readily releasable with respect to the intrinsic $\mathrm{Ca}^{2+}$ sensitivity. Their release probability in response to single APevoked $\left[\mathrm{Ca}^{2+}\right]$ transients is negligible but increases gradually during consecutive APs (see Results).

In conclusion, we find that the developmental specialization of the calyx toward high-fidelity, high-frequency transmission may be aided by a spatial reorganization of its release sites toward the proposed nonuniform topography.

\section{REFERENCES}

Adler EM, Augustine GJ, Duffy SN, Charlton MP (1991) Alien intracellular calcium chelators attenuate neurotransmitter release at the squid giant synapse. J Neurosci 11:1496-1507.

Albritton NL, Meyer T, Stryer L (1992) Range of messenger action of calcium ion and inositol 1,4,5-triphosphate. Science 258:1812-1815.

Auger C, Marty A (2000) Quantal currents at single-site central synapses. J Physiol (Lond) 526:3-11.

Augustine GJ (2001) How does calcium trigger neurotransmitter release? Curr Opin Neurobiol 11:320-326.

Augustine GJ, Neher E (1992) Neuronal $\left[\mathrm{Ca}^{2+}\right]$ signaling takes the local route. Curr Opin Neurobiol 2:302-307.

Augustine GJ, Adler EM, Charlton MP (1991) The calcium signal for transmitter secretion from presynaptic nerve terminals. Ann NY Acad Sci 635:365-381.

Barnes-Davies M, Forsythe ID (1995) Pre- and postsynaptic glutamate receptors at a giant excitatory synapse in rat auditory brainstem slices. J Physiol (Lond) 488:387-406.

Baylor SM, Hollingworth S (1998) Model of sarcomeric $\mathrm{Ca}^{2+}$ movements, including ATP $\mathrm{Ca}^{2+}$ binding and diff usion, during activation of frog skeletal muscle. J Gen Physiol 112:297-316.

Bennett MR, Farnell L, Gibson WG (2000) The probability of quantal secretion within an array of calcium channels of an active zone. Biophys J 78:2222-2240.

Bertram R, Smith GD, Sherman A (1999) Modeling study of the effects of overlapping $\mathrm{Ca}^{2+}$ microdomains on neurotransmitter release. Biophys J 76:735-750.

Blank PS, Cho MS, Vogel SS, Kaplan D, Kang A, Malley J, Zimmerberg $\mathrm{J}$ (1998) Submaximal responses in calcium-triggered exocytosis are explained by differences in the calcium sensitivity of individual secretory vesicles. J Gen Physiol 112:559-567.

Bollmann JH, Sakmann B, Borst JGG (2000) Calcium sensitivity of glutamate release in a calyx-type terminal. Science 289:953-957.

Borst JGG, Sakmann B (1996) Calcium influx and transmitter release in a fast CNS synapse. Nature 383:431-434.

Borst JGG, Sakmann B (1998) Calcium current during single action potential in a large presynaptic terminal of the rat brain stem. J Physiol (Lond) 506:143-157.

Borst JGG, Sakmann B (1999a) Effects of changes in action potential shape on calcium currents and transmitter release in a calyx-type synapse of the rat auditory brain stem. Philos Trans R Soc Lond B Biol Sci 354:347-355.

Borst JGG, Sakmann B (1999b) Depletion of calcium in the synaptic cleft of a calyx-type synapse in the rat brainstem. J Physiol (Lond) 521:123-133.

Borst JGG, Helmchen F, Sakmann B (1995) Pre- and postsynaptic whole-cell recordings in the medial nucleus of the trapezoid body of the rat. J Physiol (Lond) 489:825-840.

Chow RH, Klingauf J, Neher E (1994) Time course of $\mathrm{Ca}^{2+}$ concentration triggering exocytosis in neuroendocrine cells. Proc Natl Acad Sci USA 91:12765-12769.

Church PJ, Stanley EF (1996) Single L-type calcium channel conductance with physiological levels of calcium in chick ciliary ganglion neurons. J Physiol (Lond) 496:59-68.

Colecraft HM, Brody DL, Yue DT (2001) G-protein inhibition of N-and P/Q-type calcium channels: distinctive elementary mechanisms and their functional impact. J Neurosci 21:1137-1147.

Cooper RL, Winslow JL, Govind CK, Atwood HL (1996) Synaptic structural complexity as a factor enhancing probability of calciummediated transmitter release. J Neurophysiol 75:2451-2466.

DiGregorio DA, Peskoff A, Vergara JL (1999) Measurement of action potential-induced presynaptic calcium domains at a cultured neuromuscular junction. J Neurosci 19:7846-7859.

Dobrunz LE, Stevens CF (1997) Heterogeneity of release probability, facilitation, and depletion at central synapses. Neuron 18:995-1008.

Fisher SA, Fischer TM, Carew TJ (1997) Multiple overlapping processes underlying short-term synaptic enhancement. Trends Neurosci 20:170-177.

Forsythe ID, Barnes-Davies M, Brew HM (1995) The calyx of Held: a model for transmission at mammalian glutamatergic synapses. In: Excitatory amino acids and synaptic transmission (Wheal $\mathrm{H}$, Thomson A, eds), pp 133-144. London: Academic.

Gil A, Segura J, Pertusa JAG, Soria B (2000) Monte Carlo simulation of $3-\mathrm{D}$ buffered $\mathrm{Ca}^{2+}$ diffusion in neuroendocrine cells. Biophys $\mathrm{J}$ 78:13-33.

Glavinovic MI, Rabie HR (2001) Monte Carlo evaluation of quantal analysis in the light of $\mathrm{Ca}^{2+}$ dynamics and the geometry of secretion. Pflügers Arch 443:132-145.

Gollasch M, Hescheler J, Quale JM, Patlak JB, Nelson MT (1992) Single calcium channel currents of arterial smooth muscle at physiological calcium concentrations. Am J Physiol 263:C948-C952.

Harlow LH, Ress D, Stoschek A, Marshall RM, McMahan UJ (2001) The architecture of active zone material at the frog's neuromuscular junction. Nature 409:479-484.

Helmchen F, Borst JGG, Sakmann B (1997) Calcium dynamics associated with a single action potential in a CNS presynaptic terminal. Biophys J 72:1458-1471.

Isaacson JS, Walmsley B (1995) Counting quanta: direct measurements of transmitter release at a central synapse. Neuron 15:875-884.

Iwasaki S, Takahashi T (1998) Developmental changes in calcium channel types mediating synaptic transmission in rat auditory brain stem. J Physiol (Lond) 509:419-423.

Katz B (1969) The release of neural transmitter substances. Springfield, IL: Thomas.

Klingauf J, Neher E (1997) Modeling buffered $\mathrm{Ca}^{2+}$ diffusion near the membrane: implications for secretion in neuroendocrine cells. Biophys J 72:674-690.

Llinãs R, Sugimori M, Silver RB (1992) Microdomains of high calcium concentration in a presynaptic terminal. Science 256:677-679.

Markram H, Lübke J, Frotscher M, Roth A, Sakmann B (1997) Physiology and anatomy of synaptic connections between thick tufted pyramidal neurones in the developing rat neocortex. J Physiol (Lond) 500:409-440.

Msghina M, Millar AG, Charlton MP, Govind CK, Atwood HL (1999) Calcium entry related to active zones and differences in transmitter release at phasic and tonic synapses. J Neurosci 19:8419-8434.

Nägerl UV, Novo D, Mody I, Vergara JL (2000) Binding kinetics of calbindin- $\mathrm{D}_{28 \mathrm{k}}$ determined by flash photolysis of caged $\mathrm{Ca}^{2+}$. Biophys J 79:3009-3018.

Naraghi M, Neher E (1997) Linearized buffered $\mathrm{Ca}^{2+}$ diffusion in microdomains and its implications for calculation of $\left[\mathrm{Ca}^{2+}\right]$ at the mouth of a calcium channel. J Neurosci 17:6961-6973.

Neher E (1986) Concentration profiles of intracellular calcium in the presence of a diffusable chelator. Exp Brain Res 14:80-96. 
Neher E (1998a) Vesicle pools and $\mathrm{Ca}^{2+}$ microdomains: new tools for understanding their roles in neurotransmitter release. Neuron 20:389-399.

Neher E (1998b) Usefulness and limitations of linear approximations to the understanding of $\mathrm{Ca}^{2+}$ signals. Cell Calcium 24:345-357.

Oertel D (1999) The role of timing in the brain stem auditory nuclei of vertebrates. Annu Rev Physiol 61:497-519.

Ohana O, Sakmann B (1998) Transmitter release modulation in nerve terminals of rat neocortical pyramidal cells by intracellular calcium buffers. J Physiol (Lond) 513:135-148.

Press WH, Teukolsky SA, Vetterling WT, Flannery BP (1988) Numerical recipes in C. New York: Cambridge UP.

Quastel DMJ, Guan YY, Saint DA (1992) The relation between transmitter release and $\mathrm{Ca}^{2+}$ entry at the mouse motor nerve terminal: role of stochastic factors causing heterogeneity. Neuroscience 51:657-671.

Rowland KC, Irby NK, Spirou AG (2000) Specialized synapseassociated structures within the calyx of Held. J Neurosci 20:9135-9144.

Rozov A, Burnashev N, Sakmann B, Neher E (2001) Transmitter release modulation by intracellular $\mathrm{Ca}^{2+}$ buffers in facilitating and depressing nerve terminals of pyramidal cells in layer $2 / 3$ of the rat neocortex indicates a target cell-specific difference in presynaptic calcium dynamics. J Physiol (Lond) 531:807-826.

Sakaba T, Neher E (2001a) Preferential potentiation of fast-releasing synaptic vesicles by cAMP at the calyx of Held. Proc Natl Acad Sci USA 98:331-336.

Sakaba T, Neher E (2001b) Quantitative relationship between transmitter release and calcium current at the calyx of Held synapse. J Neurosci 21:462-476.

Schneggenburger R, Neher E (2000) Intracellular calcium dependence of transmitter release rates at a fast central synapse. Nature 406:889-893.

Schneggenburger R, Meyer AC, Neher E (1999) Released fraction and total size of pool of immediately available transmitter quanta at a calyx synapse. Neuron 23:399-409.
Silver RA, Momiyama A, Cull-Candy SG (1998) Locus of frequencydependent depression identified with multiple-probability fluctuation analysis at rat climbing fibre-Purkinje cell synapses. J Physiol (Lond) 510:881-902.

Smith GD (2001) Modeling local and global calcium signals using reaction diffusion equations. In: Computational neuroscience (Schutter ED ed), pp 49-85. Boca Raton, FL: CRC.

Stanley EF (1997) The calcium channel and the organization of the presynaptic transmitter release face. Trends Neurosci 20:404-409.

Sun J, Wu L (2001) Fast kinetics of exocytosis revealed by simultaneous measurements of presynaptic capacitance and postsynaptic currents at a central synapse. Neuron 30:171-182.

Taschenberger H, von Gersdorff H (2000) Fine-tuning an auditory synapse for speed and fidelity: developmental changes in presynaptic waveform, EPSC kinetics, and synaptic plasticity. J Neurosci 20:9162-9173

Van der Kloot W (1988) Estimating the timing of quantal releases during end-plate currents at the frog neuromuscular junction. J Physiol (Lond) 402:595-603.

Wang LY, Kaczmarek LK (1998) High-frequency firing helps replenish the readily releasable pool of synaptic vesicles. Nature 394:384-388.

Wu LG, Borst JGG (1999) The reduced release probability of releasable vesicles during recovery from short-term synaptic depression. Neuron 23:821-832.

Wu LG, Westenbroek RE, Borst JGG, Catterall WA, Bert Sakmann (1999) Calcium channel types with distinct presynaptic localization couple differentially to transmitter release in single calyx-type synapses. J Neurosci 19:726-736.

Yamada WM, Zucker RS (1992) Time course of transmitter release calculated from simulations of a calcium diffusion model. Biophys $\mathrm{J}$ 61:671-682.

Yoshikami D, Bagabaldo Z, Olivera BM (1989) The inhibitory effects of omega-conotoxins on Ca channels and synapses. Ann NY Acad Sci 560:230-248. 